\title{
pH-Responsive Nanocomposite Based Hydrogels for the Controlled Delivery of Ticagrelor; In Vitro and In Vivo Approaches
}

\author{
Nariman Shahid ${ }^{1,2}$ \\ Alia Erum' \\ Muhammad Zaman ${ }^{3}$ \\ Ume Ruqia Tulain' \\ Qurat-ul-ain Shoaib ${ }^{3}$ \\ Abdul Majeed (D) 4 \\ Muhammad F Rasool (iD) ${ }^{4}$ \\ Imran Imran ${ }^{5}$ \\ Sultan Alshehri (iD ${ }^{6}$ \\ Behnam Noorani ${ }^{7}$ \\ Faleh Alqahtani $\mathbb{D}^{8}$ \\ 'College of Pharmacy, University of \\ Sargodha, Sargodha, 40100, Pakistan; \\ ${ }^{2}$ Akhtar Saeed College of Pharmaceutical \\ Sciences, Lahore, 53720, Pakistan; \\ ${ }^{3}$ Faculty of Pharmacy, University of \\ Central Punjab, Lahore, 54782, Pakistan; \\ ${ }^{4}$ Department of Pharmacy Practice, \\ Faculty of Pharmacy, Bahauddin Zakariya \\ University, Multan, 60800, Pakistan; \\ ${ }^{5}$ Department of Pharmacology, Faculty of \\ Pharmacy, Bahauddin Zakariya University, \\ Multan, 60800, Pakistan; ${ }^{6}$ Department of \\ Pharmaceutics, College of Pharmacy, King \\ Saud University, Riyadh, II45I, Saudi \\ Arabia; ${ }^{7}$ Department of Pharmaceutical \\ Sciences, Jerry H. Hodge School of \\ Pharmacy, Texas Tech University Health \\ Sciences Center, Amarillo, TX, 79106, \\ USA; ${ }^{8}$ Department of Pharmacology and \\ Toxicology, College of Pharmacy, King \\ Saud University, Riyadh, II45I, Saudi \\ Arabia
}

Correspondence: Muhammad Zaman; Faleh Alqahtani

Email dr.mzaman@ucp.edu.pk;

afaleh@ksu.edu.sa
Background: Ticagrelor (TG), an antiplatelet drug is employed to treat patients with acute coronary syndrome, but its inadequate oral bioavailability due to poor solubility and low permeability restricts its effectiveness.

Purpose: This contemporary work was aimed to design a novel $\mathrm{pH}$-sensitive nanocomposite hydrogel $(\mathrm{NCH})$ formulation incorporating thiolated chitosan $(\mathrm{TCH})$ based nanoparticles (NPs) of Ticagrelor (TG), to enhance its oral bioavailability for effectively inhibiting platelet aggregation.

Methods: NCHs were prepared by free radical polymerization technique, using variable concentrations of chitosan $(\mathrm{CH})$ as biodegradable polymer, acrylic acid (AA) as a monomer, $\mathrm{N}, \mathrm{N}$-methylene bisacrylamide (MBAA) as cross-linker, and potassium persulphate (KPS) as initiator.

Results: The optimum hydrogel formulation was selected for fabricating NCHs, considering porosity, sol-gel fraction, swelling studies, drug loading capacity, and TG's in vitro release as determining factors. Outcomes of the studies have shown that the extent of hydrogel swelling and drug release was comparatively greater at higher $\mathrm{pH}$ (7.4). Moreover, an amplifying trend was observed for drug loading and hydrogel swelling by increasing AA content, while it declined by increasing MBAA. The NCHs were evaluated by various physicochemical techniques and the selected formulation was subjected to in vivo bioavailability studies, confirming enhancement of bioavailability as indicated by prolonged half-life and multifold increase in area under the curve (AUC) as compared to pure TG.

Conclusion: The results suggest that $\mathrm{NCHs}$ demonstrated a $\mathrm{pH}$-responsive, controlled behavior along with enhanced bioavailability. Thus NCHs can be effectively utilized as efficient delivery systems for oral delivery of TG to reduce the risk of myocardial infarction. Keywords: nanocomposite, thiolated chitosan, biodegradable, bioavailability, free radical polymerization

\section{Introduction}

In pharmaceutical drug discovery and development, the poor bioavailability of drug moiety due to low aqueous solubility and permeability is a crucial obstacle limiting its effectiveness. Oral administration of hydrophobic drugs remains a challenge due to susceptibility to variable physiological $\mathrm{pH}$ and gastrointestinal enzymes, as well as low permeability of the intestinal epithelial membrane. The drugs belonging to Biopharmaceutics Classification System (BCS) class II and IV face bioavailabilityrelated issues due to retarded absorption. The improvement of the physicochemical 
characteristics of active pharmaceutical ingredients (APIs) possessing limited bioavailability is of paramount importance in this regard. ${ }^{1}$ For this rationale, much effort has been put into developing various novel nanotechnology-based drug delivery systems to improve oral availability by increasing drug residence time and enhancing solubility. In the recent era, nanotechnology has evolved as a booming strategy for enhancing the bioavailability of BCS class IV candidates. Accordingly, various approaches have been investigated to meet such challenges. Numerous innovative drug delivery technologies that have been explored in recent years for this purpose include micellar technologies, lipid-based delivery systems, size reduction techniques, complexation, hydrotropy, solid dispersion systems, porous microparticle, and nanoparticle technologies, co-crystallisation, microemulsification, supercritical fluid (SCF) process, solvent deposition, etc. ${ }^{2}$ Research trends are currently focused on the incorporation of various nanoparticulate systems such as polymeric nanoparticles, ceramic nanoparticles, carbon-based nanomaterials, and metal/metaloxide nanoparticles into the hydrogel framework to eventually obtain nanocomposite hydrogels $(\mathrm{NCH})$. This hybridization can result in a synergetic property enhancement of each component, ie the mechanical strength provided by the hydrogel and decreased aggregation of the concomitant nanoparticles. ${ }^{3}$ Such amalgamation not only helps to increase the solubility but ultimately can enhance the bioavailability of the drug. ${ }^{4}$ These hybrid hydrogels act as useful platforms for drug delivery as well as gene delivery, being intelligent, promising, and smart drug delivery devices. NCHs act as useful vehicles for targeting drugs to specific sites along with their capability of drug release in a controlled manner. $\mathrm{NCHs}$ can serve as a reservoir of drug molecules, in local drug delivery, based on the interactions between drug molecules and nano-filler and hydrogel matrix. This ability arises from the stimuli-responsiveness of these innovative composite systems. Various stimuli such as enzymatic, hydrolytic, or environmental often serve to utilize them for drug release at the desirable site. ${ }^{5}$ Stimuli-responsive $\mathrm{NCHs}$ hold promising potential for drug delivery, as the drug can be intelligently released from the gel matrix under external stimuli, such as magnetic field, electric field, $\mathrm{pH}$, temperature, etc. As a new generation of materials, bionanocomposites combine the properties of biopolymers along with the nanostructured materials and are attracting wide interest due to having potential in vitro and in vivo biomedical applications to meet new challenges such as implants' design, wound healing, drug delivery systems, bone regeneration, as tissue engineering scaffolds, and the sustained release of drugs. ${ }^{6}$ This is mainly due to their biocompatible nature, high versatility, stress absorbing potentiality, highly tunable properties, and similarity of their physical properties to natural tissues. ${ }^{7}$ Traditional hydrogels usually hold inferior mechanical qualities as well as lack in multi-functionalities. Nano-sized particles, both inorganic and organic/polymeric NPs, comprised of biodegradable polymers and inorganic clays, etc. have been intensively studied as biofunctional substances due to having unique chemical, physical, and biological attributes. The nanocomposite is a binary system comprised of a blend of materials possessing diverse properties to achieve the desired improvement in characteristics such as electric, catalytic, optical, and chemical as well as thermal stabilities. Various innovative composite hydrogels have been developed by some other researchers as well, and have been summarized in Table 1, along with a comparison of activities and applications of newly developed nanocomposite hydrogel systems.

Ticagrelor (TG), an anticoagulant drug that acts as a reversible antagonist of P2Y12 platelet receptors, has been approved for utilization in patients with acute coronary syndrome. TG, being a BCS class IV candidate, possesses very low aqueous solubility of $<10 \mathrm{ug} / \mathrm{mL}$ at all physiological $\mathrm{pH}$ levels and poor intestinal permeability, resulting in its low absolute bioavailability of $\sim 36 \%{ }^{8}$ To overcome such issues a nanocomposite hydrogel ( $\mathrm{NCH} 2)$ formulation based upon thiolated chitosan containing nanoparticles (TCH-NPs) was fabricated. ${ }^{9}$ Chitosan $(\mathrm{CH})$ is a well-known biodegradable and biocompatible polymer used widely in fabricating hydrogels. $\mathrm{CH}$ possesses additional advantages of having specific activities such as antibacterial, anti-tumor, and antioxidant. ${ }^{10}$ Chitosan derivatives have been investigated as absorption enhancers, to overcome problems such as CH's incomplete solubility and effectiveness at neutral $\mathrm{pH}^{1}$ Thiolation of $\mathrm{CH}$ results in the improved mucoadhesive potential that helps it to adhere tightly to mucosal epithelium via thiol bisulphide linkages, ultimately enhancing its cellular permeation. The physicochemical properties of TG make it a suitable candidate to be formulated as a nanoparticle and nanocomposite delivery system, which may result in producing an ameliorated and prolonged anticoagulant effect. ${ }^{11}$

Various TG formulations have been developed in past years such as conventional film-coated tablets (Brilinta, Brilique), Self-microemulsifying drug delivery systems, ${ }^{12}$ co-crystals of TG with nicotinamide, and nanolipid carriers (NLCs) for enhancing the dissolution and bioavailability of TG. ${ }^{13}$ It was reported that NLCs demonstrated a $254.9 \%$ increase in bioavailability as compared to pure TG. The currently developed novel nanocomposite hydrogel formulation will not only increase solubility due to the presence of 
Table I List of Nanocomposite Hydrogels Along with Their Applications

\begin{tabular}{|c|c|c|c|c|}
\hline Sr. No. & Nanocomposite Hydrogels & Methodology & Applications & Ref. \\
\hline I & $\begin{array}{l}\text { Alginate hydrogel-polyvinyl alcohol/silk } \\
\text { fibroin/magnesium hydroxide }\end{array}$ & $\begin{array}{l}\text { Crosslinking magnesium } \\
\text { hydroxide nanorods (NPs) }\end{array}$ & $\begin{array}{l}\text { Antibacterial, scaffold for use in wound dressing, } \\
\text { tissue engineering, and drug delivery systems. }\end{array}$ & {$[27]$} \\
\hline 2 & $\begin{array}{l}\text { Core-shell magnetic nanocomposite } \\
\text { based on cross-linked chitosan hydrogels }\end{array}$ & Chemical cross-linking & $\begin{array}{l}\text { Used for in vitro hyperthermia of cancer therapy, } \\
\text { Highly stable and porous }\end{array}$ & [28] \\
\hline 3 & $\begin{array}{l}\text { Chitosan/pyromellitimide benzoyl } \\
\text { thiourea hydrogels }\end{array}$ & Chemical cross-linking & Antimicrobial activity, Super porosity & [29] \\
\hline 4 & $\begin{array}{l}\text { Hybrid monodispersed magnetic } \alpha- \\
\mathrm{Fe}_{2} \mathrm{O}_{3} \text { based chitosan nanocomposite } \\
\text { film }\end{array}$ & $\begin{array}{l}\text { Dispersion of magnetic } \alpha- \\
\text { Fe2O3 nanoparticles }\end{array}$ & Industrial and biomedical applications & {$[30]$} \\
\hline 5 & $\begin{array}{l}\text { Chitosan/hydroxyapatite composite } \\
\text { nanofiber }\end{array}$ & Electrospinning process & $\begin{array}{l}\text { Removal of heavy metal ions, Industrial } \\
\text { applications }\end{array}$ & {$[31]$} \\
\hline 6 & $\begin{array}{l}\text { Nanocomposite based on magnetic } \\
\text { dopamine nanoparticles }\end{array}$ & Dispersion technique & Purification of $\alpha$-amylase from the bovine milk & {$[32]$} \\
\hline 7 & $\begin{array}{l}\text { Hyaluronic acid-based nanocomposite } \\
\text { hydrogels }\end{array}$ & $\begin{array}{l}\text { Photopolymerization } \\
\text { method }\end{array}$ & Ocular drug delivery applications & {$[33]$} \\
\hline \multicolumn{5}{|c|}{ Recent Research work } \\
\hline 8 & $\begin{array}{l}\text { Nanocomposite hydrogels based on } \\
\text { thiolated chitosan nanoparticles }\end{array}$ & $\begin{array}{l}\text { Chemical cross-linking } \\
\text { (Free radical } \\
\text { polymerization) }\end{array}$ & $\begin{array}{l}\text { Oral delivery system TG with enhanced } \\
\text { bioavailability } \\
\text { Enhanced anticoagulant effect } \\
\text { Biocompatible and biodegradable drug delivery } \\
\text { system } \\
\text { Sustained and controlled drug release } \\
\text { pH-responsive swelling } \\
\text { Highly stable and porous networks }\end{array}$ & $\begin{array}{l}\text { Current } \\
\text { study }\end{array}$ \\
\hline
\end{tabular}

nanoparticles but will also enhance the permeability of $\mathrm{TG}$ owing to the presence of thiolated chitosan. The small size of the NPs provides a large surface area for prompt dissolution and absorption of the drug through the intestinal membrane, enhancing the bioavailability and anti-platelet activity of TG. An additional advantage of this hybrid nanocomposite network might be the sustained and controlled release of TG, promoting a reduction in dosing frequency and ultimately increasing patient compliance. ${ }^{14}$

\section{Materials and Methods}

\section{Materials}

Ticagrelor (TG) was provided as a gift from CCL Pharmaceuticals Pvt. Ltd., Lahore, Pakistan. Low molecular weight chitosan (CH) (100 kDa, 20-300 cP viscosity, 75$85 \%$ deacetylation), acrylic acid, acetonitrile, ammonium hydroxide, ammonium acetate, dithio-bis nitrobenzoic acid (Ellman's reagent), ethyl 3-(3-dimethyl aminopropyl) carbodiimide hydrochloride (EDAC), ethanol, methylene-bis acrylamide (MBAA), methanol, potassium persulphate (KPS) and sodium tripolyphosphate (TPP) were purchased from Sigma-Aldrich, USA. Glacial acetic acid (GAA), sodium hydroxide $(\mathrm{NaOH})$, sodium dihydrogen phosphate $\left(\mathrm{NaH}_{2} \mathrm{PO}_{4}\right)$ and, thioglycolic acid (TGA) were purchased from Merck Darmstadt, Germany.

Sodium chloride $(\mathrm{NaCl})$ and hydrochloric acid $(\mathrm{HCl})$ were purchased from Supelco, USA. Distilled water was acquired from the research laboratory of The University of Lahore. All the chemicals and reagents utilized were of analytical grade.

\section{Method}

\section{Thiolation of Chitosan}

Thiolation of $\mathrm{CH}$ was carried out by a previously reported coupling reaction..$^{10}$ Briefly, $\mathrm{CH}(500 \mathrm{mg})$ was dissolved in $50 \mathrm{~mL}$ of $1 \%$ acetic acid solution. $100 \mathrm{mg}$ of ethyl 3-(3-dimethyl aminopropyl) carbodiimide hydrochloride (EDAC), as a catalyst, was added in $\mathrm{CH}$ solution (to make a final concentration of $125 \mathrm{mM}$ ). Thereafter $500 \mathrm{mg}$ of 
thioglycolic acid (TGA) was added upon proper mixing of EDAC, and the $\mathrm{pH}$ of the medium was adjusted at 5, using 1 $\mathrm{mM} \mathrm{HCl}$ solution to avoid disulfide bond formation among polymer chains. This reaction mixture was incubated at room temperature in darkness for 4 hours, under constant stirring. Afterward, the polymer solution was dialyzed (dialysis membrane of molecular weight cut off $12 \mathrm{kDa}$ ) in darkness against $5 \mathrm{mM} \mathrm{HCl}$ for 3 days to remove unbound TGA and to isolate polymer conjugates. The polymer solution was again dialyzed against $5 \mathrm{mM} \mathrm{HCl}$ containing $1 \% \mathrm{NaCl}$ for 2 days, to eliminate interactions between the cationic polymer and anionic sulfhydryl groups. After dialysis, the samples were frozen at $-50{ }^{\circ} \mathrm{C}$ and lyophilized. The freeze-dried samples were stored in air-tight containers for further use. Thiolation of $\mathrm{CH}$ has been demonstrated in Figure 1. Thiolated $\mathrm{CH}$ was characterized by FT-IR to confirm thiol group substitution and the formation of an amide bond. Furthermore, thiol content was also estimated by Ellman's reagent. ${ }^{11}$

\section{Preparation of TG Loaded Nanoparticles}

Both chitosan nanoparticles (CH-NPs) and thiolated chitosan nanoparticles (TC-NPs) loaded with TG were prepared following ionic gelation technique, using sodium tripolyphosphate (TPP) as a crosslinker, as previously reported by Tan Vui Nguyan and Reynaldo Esquivel. Various formulations were attempted by varying concentrations of $\mathrm{CH}$ concerning TPP and TG in preliminary screening. $\mathrm{CH}$ and TC were dissolved in $2 \% \mathrm{v} / \mathrm{v}$ acetic acid solution separately and left stirring overnight. TG solution $(5 \mathrm{mg} / \mathrm{mL})$ was added dropwise in polymer solutions. The $\mathrm{pH}$ of the solution was adjusted at 4.5 and was left stirring for 20 minutes. Thereafter, TPP solution having a concentration of $3 \mathrm{mg}$ per $\mathrm{mL}(0.3 \%)$ was added dropwise and constantly stirred until the opalescent appearance of the solution. Thereafter samples were ultracentrifuged at $12,000 \mathrm{rpm}$ for 0.5 hours. Sedimented nanoparticles were frozen and lyophilized at $-50^{\circ} \mathrm{C}$ and then stored safely in capped containers. ${ }^{15}$

\section{Characterization of TG Loaded NPs}

TG-loaded NPs were evaluated by physicochemical characterizations such as FT-IR, SEM, XRD, zeta potential, particle size, entrapment efficiency, and loading capacity to ensure ample drug loading. ${ }^{13}$
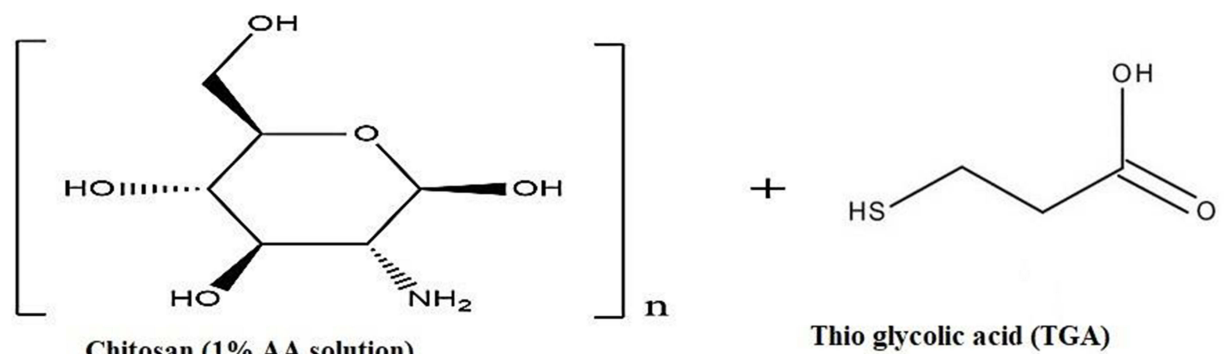

Chitosan (1\% AA solution)

Thio glycolic acid (TGA)

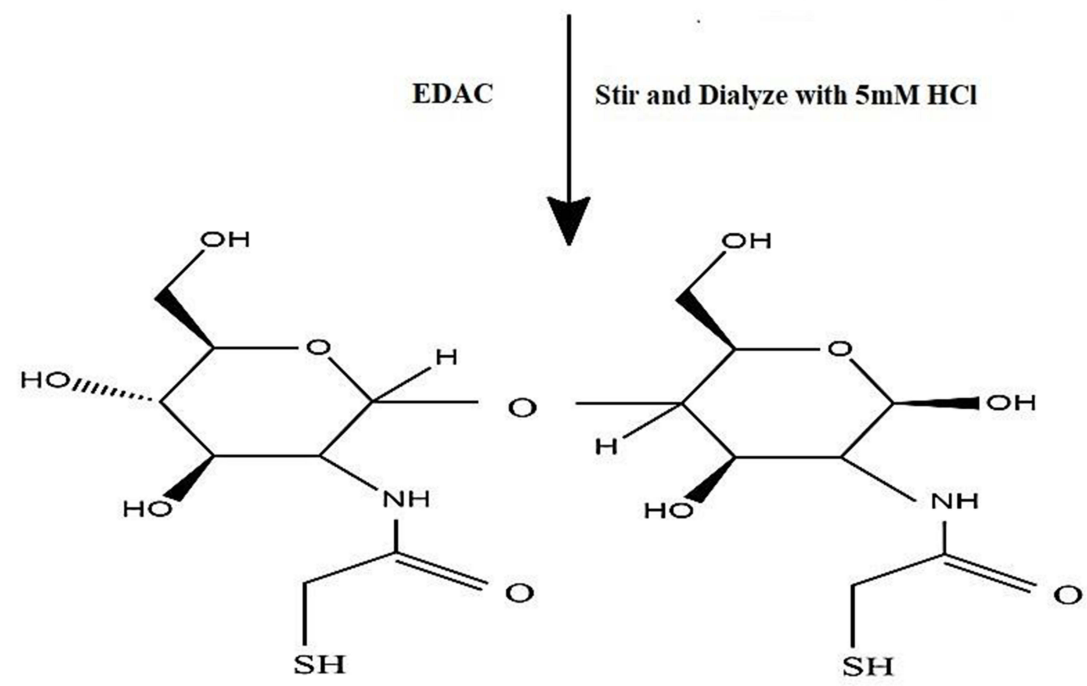

Chitosan-Thioglycolic acid congugate (TCH)

Figure I Reaction scheme of chitosan-thioglycolic acid conjugate (TCH). 


\section{Preparation of Nanocomposite Hydrogels ( $\mathrm{NCHs)}$}

Various hydrogel formulations were sought in the initial trial phase by varying polymer $(\mathrm{CH})$, monomer (AA), and crosslinker (MBAA) content, adopting the free radical polymerization technique. $\mathrm{CH}$ was dissolved in $2 \% \mathrm{v} / \mathrm{v}$ acetic acid (AA) solution with continuous stirring at a speed of 250 to $300 \mathrm{rpm}$ at $70{ }^{\circ} \mathrm{C}$. Potassium persulphate (KPS) was added as a polymer initiator in the above solution. Methylene bis acrylamide (MBAA), as a crosslinking agent was mixed up in an acrylic acid (AA) monomer and added to the above solution. Test tubes containing sample solution were incubated at $60-70{ }^{\circ} \mathrm{C}$ in a water bath for up to 24 hours. Hydrogel discs were cut and washed with $30 \% \mathrm{v} / \mathrm{v}$ ethanol solution, after 24 hours to remove any surplus material (unreacted monomer) and to neutralize $\mathrm{pH}$ as well. Hydrogel discs were then dried at $45-50{ }^{\circ} \mathrm{C}$ and stored in airtight containers for further characterization and use. ${ }^{16}$ The composition of various hydrogel formulations is presented in Table 2 and the scheme for the preparation of nanocomposite hydrogels (NCHs) has been displayed in Figure 2.

\section{In Vitro Characterization of Hydrogels Porosity Measurement}

To determine hydrogel's porosity, a solvent replacement method was adopted. Hydrogel discs of all formulations were submerged overnight in ethanol and weighed after mopping extra ethanol from the surface. The following equation was used to calculate the porosity

$$
\text { Porosity }=M 2-M 1 / \rho M_{2}-M_{1} V * 100
$$

where, $M_{1}$ and $M_{2}$ are the hydrogel mass before and after ethanol immersion, respectively, $\rho$ is the density of absolute alcohol, whereas, $\mathrm{V}$ is the hydrogel's volume. ${ }^{17}$

Table 2 Composition of Hydrogels $(\mathrm{HI}-\mathrm{H} 9)$

\begin{tabular}{|l|c|c|c|c|}
\hline Formulation & $\begin{array}{c}\text { Polymer } \\
\text { (\%w/v) }\end{array}$ & $\begin{array}{c}\text { Monomer } \\
\text { (\%w/v) }\end{array}$ & $\begin{array}{c}\text { Cross } \\
\text { Linker } \\
\text { (\%w/v) }\end{array}$ & $\begin{array}{c}\text { Initiator } \\
\text { (\%w/v) }\end{array}$ \\
\hline HI & 0.3 & 15 & 0.3 & 0.2 \\
H2 & 0.5 & 15 & 0.3 & 0.2 \\
H3 & 0.7 & 15 & 0.3 & 0.2 \\
H4 & I & 10 & 0.3 & 0.2 \\
H5 & I & 12.5 & 0.3 & 0.2 \\
H6 & I & 15 & 0.3 & 0.2 \\
H7 & I & 15 & 0.4 & 0.2 \\
H8 & I & 15 & 0.5 & 0.2 \\
H9 & I & 15 & 0.6 & 0.2 \\
\hline
\end{tabular}

Abbreviation: $\mathrm{HI}-\mathrm{H} 9$, various hydrogel formulations.

\section{Determination of Sol-Gel Fraction}

Pre-weighed hydrogel discs of various formulations were immersed for 48 hours in distilled water at ambient temperature. An adequate quantity of distilled water was taken to ensure proper submersion. After 48 hours, discs were dried in an oven at $45^{\circ} \mathrm{C}$ until constant weight. Gel and sol fractions were calculated using the below-mentioned equations.

$$
\begin{gathered}
\text { Gel fraction }=(\mathrm{Wd} / \mathrm{Wi}) \times 100 \\
\text { Solfraction }=100-\text { Gel Fraction }
\end{gathered}
$$

Where Wi is the initial weight of the dried sample and $\mathrm{Wd}$ is the weight of the dried sample after extraction with water. ${ }^{16}$

\section{Swelling Studies}

Dynamic and equilibrium swelling studies were performed at physiological $\mathrm{pH} \mathrm{1.2,4.5,6.8} \mathrm{and} \mathrm{7.4,} \mathrm{to} \mathrm{evaluate} \mathrm{the}$ $\mathrm{pH}$ sensitivity of prepared hydrogel formulations. Swelling ratio (q) and percentage equilibrium swelling (\%ES) was calculated as follow:

$$
\begin{gathered}
q=M s / M o \\
\% E S=M e q-M o / M e q * 100
\end{gathered}
$$

Where Ms is the swollen hydrogel's mass at the time $(\mathrm{t})$, Mo is the initial mass of dried hydrogel disc and Meq is the swollen hydrogel's mass at the time of equilibrium. ${ }^{16,17}$

\section{Drug Loading and Entrapment Efficiency}

Pre-weighed discs were immersed separately for 48 hours in $50 \mathrm{~mL}(1 \% \mathrm{w} / \mathrm{v})$ TG solution ( $\mathrm{pH} \mathrm{7.4)}$. After 48 hours, discs were removed, washed with deionized water, and dried in the oven at $50{ }^{\circ} \mathrm{C}$ until constant weight. The same procedure was adopted for loading hydrogels with TG-containing NPs. Drug loading was determined by both the weight method and by extraction method.

For the weight method, the initial weight of the unloaded disc was subtracted from the weight of the dried loaded disc using the formula:

$$
\begin{gathered}
\text { Amount loaded }=W_{a}-W_{b} \\
\text { Drug loading } \% \text { age }=\left[W_{a}-W_{b} / W_{b}\right] * 100
\end{gathered}
$$

Where, $\mathrm{W}_{\mathrm{a}}$ and $\mathrm{W}_{\mathrm{b}}$ are the weights of dried discs after and before drug loading, respectively.

In the extraction method, repeated extraction was done using $25 \mathrm{~mL}$ fresh deionized water each time, until no drug concentration was observed in the last extracted 


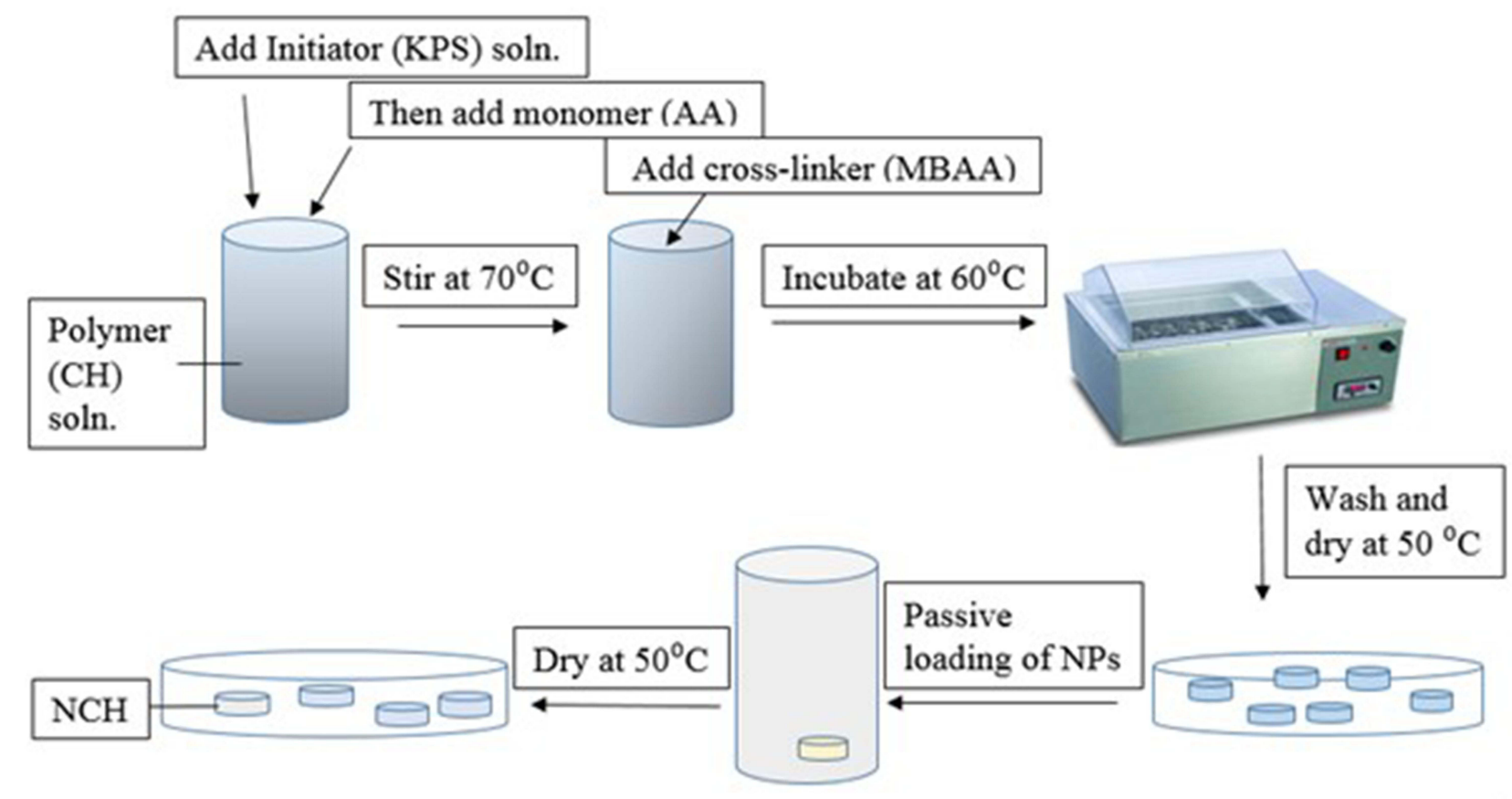

Figure 2 Schematic diagram for preparation of nanocomposite hydrogels $(\mathrm{NCHs})$.

sample. Drug concentration was spectrophotometrically determined at $254 \mathrm{~nm}(\lambda \max ) .{ }^{16,17}$

\section{FT-IR Spectroscopy}

Fourier transform infrared (FT-IR) spectra of TG, TG loaded hydrogel (DLH), nanocomposite hydrogels containing $\mathrm{CH}-\mathrm{NPs}(\mathrm{NCH} 1)$ and nanocomposite hydrogels containing TC-NPs (NCH2) were scanned by FT-IR spectrometer ALPHA (Bruker, Germany). Powdered samples were placed on the sample handler and were scanned over the range of $4000-500 \mathrm{~cm}^{-1}$ at ambient temperature. ${ }^{17}$

\section{Morphological Analysis/Scanning Electron Microscopy (SEM)}

The surface morphology of hydrogels was observed by scanning electron microscope (JSM-6480LV, JEOL, Japan). Hydrogel discs were fixed on the platinum stub in a vacuum and were examined under an electron microscope at various magnifications. ${ }^{17}$

\section{Diffraction Scanning Calorimetry (DSC)}

To evaluate energy changes in TG, unloaded hydrogel, TG loaded CH-NPs, TG loaded TC-NPs, TG loaded hydrogel (NCH2) and physical mixture, thermal analysis was conducted by DSC, utilizing thermal analyzer (Q2000 V24, DSC, Seoul, Korea). A 5-10 mg sample was placed in each aluminum pan and was covered tightly with a lid. The samples were subjected to heating under nitrogen flow $(20 \mathrm{~mL} / \mathrm{min})$, at $30-310^{\circ} \mathrm{C}$. Thermograms were recorded adjusting the heating rate at $20{ }^{\circ} \mathrm{C}$ per minute. ${ }^{13}$

\section{Powder X-Ray Diffraction (PXRD) Analysis}

An X-ray diffractometer was used to demonstrate structural changes in pure TG, TG loaded hydrogel (DLH), and nanocomposite hydrogels ( $\mathrm{NCH} 1$ and $\mathrm{NCH} 2)$. XRD was carried out at an angle of $5^{\circ}$ to $80^{\circ}$ at $2 \theta$. For continuous scan mode, a $0.02^{\circ} / \mathrm{s}$ step size was set. ${ }^{17}$

\section{In Vitro Drug Release}

In vitro release studies were performed at $37{ }^{\circ} \mathrm{C}$, using USP dissolution apparatus II (75 rpm), utilizing USP buffers of $\mathrm{pH} 1.2$ and 7.4 as dissolution media. Each disc was immersed in $900 \mathrm{~mL}$ acidic buffer (pH1.2) for $2 \mathrm{~h}$, and then in $900 \mathrm{~mL}$ phosphate buffer (pH7.4) for observing drug release up to $24 \mathrm{~h}$. About $5 \mathrm{~mL}$ samples were withdrawn after specific time intervals $(0.5,1,2,4,6,8,12,24 \mathrm{~h})$ and replaced with fresh medium every time. TG release was determined spectrophotometrically at $255 \mathrm{~nm}(\lambda \max )$. DD solver software was utilized for data modeling. ${ }^{17}$

\section{Acute Oral Toxicity Studies}

An acute oral toxicity study was conducted as per OECD guidelines (423) on albino rabbits which were divided into 
three groups $(\mathrm{n}=3)$. Healthy female rabbits weighing about $1.5 \pm 0.5 \mathrm{~kg}$ were selected and kept at ambient conditions (room temperature of $25 \pm 2{ }^{\circ} \mathrm{C}$, relative humidity of $65 \pm 5 \%$, and light/dark cycle of $12 \mathrm{~h}$ ) and provided with food and water. ${ }^{18}$ Group 1 was kept as a control group while groups 2 and 3 were administered an oral dose of $5000 \mathrm{mg} / \mathrm{Kg}$ body weight, as LD50 of TG in rabbits is greater than $2000 \mathrm{mg}$. The animal study was approved by the Research and ethics committee of The University of Lahore. The NCHs were administered by oral gavage after moistening with water. After dose administration, rabbits were observed for the first $4 \mathrm{~h}$ and later after the 3rd, 7th and 14th day for any signs of toxicity (discomfort, activity, morbidity, and mortality). After 14 days the animals were sacrificed. Blood samples were taken in EDTA tubes for hematological evaluation. Vital organs such as kidney, heart, lungs, liver, brain, stomach, intestines were removed, weighed, preserved in $10 \%$ formaldehyde solution (buffered) and, later stained slides of segmented parts were prepared for histopathology. ${ }^{19}$

\section{In Vivo Bioavailability Study in Rabbits Animal Study}

The pharmacokinetic study was carried out in albino rabbits to compare the bioavailability of pure TG with that of TG-NPs and TC-NPs loaded nanocomposite hydrogels, $\mathrm{NCH} 1$ and $\mathrm{NCH} 2$, respectively. All experimental protocols were conducted in accordance with the "Guiding Principles in the Use of Animals in Toxicology" employed by the Society of Toxicology (Reston, USA). The study was approved by the Pharmacy Research Ethics Committee (PREC), University of Lahore, Pakistan Vide ref. no. IREC2017.142A. Fifteen albino rabbits, weighing about 1.5$2 \mathrm{~kg}$ were divided into three groups and were administered an oral dose of $10 \mathrm{mg} / \mathrm{kg}$ each. Group A was administered pure TG $(10 \mathrm{mg} / \mathrm{kg})$, groups $\mathrm{B}$ and $\mathrm{C}$ were administered $\mathrm{NCH} 1$ and $\mathrm{NCH} 2$, respectively, by oral gavage at an equivalent dose. Blood samples were collected from the orbital sinus at $0.5,1,2,4,6,8,12$ and $24 \mathrm{~h}$ intervals, in microfuse tubes which were then ultracentrifuged. Plasma samples were frozen at $-80{ }^{\circ} \mathrm{C}$ in an ultra-low freezer (Arctiko, Denmark) for further analysis. $^{13}$

\section{Plasma Sample Analysis}

The $0.5 \mathrm{~mL}$ plasma samples were diluted with $1 \mathrm{~mL}$ methanol, vortexed and ultracentrifuged $(15,000 \mathrm{rpm})$ for
$10 \mathrm{~min}$. The supernatant was dried at $45^{\circ} \mathrm{C}$, reconstituted with $0.5 \mathrm{~mL}$ mobile phase, filtered by $0.45 \mu \mathrm{m}$ membrane filters and, analyzed by HPLC. ${ }^{14}$

\section{HPLC Analysis}

For analysis of samples by high-performance liquid chromatography (HPLC), a previously reported method was slightly modified. A composition of 55:45 was used for the mobile phase, comprising acetonitrile and $50 \mathrm{mM}$ ammonium acetate buffer. The mobile phase was filtered $(0.45$ $\mu \mathrm{m}$ membrane filter), degassed and $\mathrm{pH}$ was adjusted to 8 using $6 \mathrm{M} \mathrm{NH}_{4} \mathrm{OH}$ solution. The flow rate of $1 \mathrm{~mL} / \mathrm{min}$, lambda max of $254 \mathrm{~nm}$, and injection volume of $20 \mu \mathrm{L}$ was adjusted. The developed method was further validated following ICH guidelines. $^{20}$

\section{Pharmacokinetic Profiling}

A calibration curve was constructed using spiked plasma samples with a known drug concentration of $0.2-50 \mu \mathrm{g} /$ $\mathrm{mL}$. PK-solver software was used to quantify pharmacokinetic parameters such as $\mathrm{t}_{1 / 2}(\mathrm{~h}), \mathrm{C}_{\max }(\mathrm{ng} / \mathrm{mL}), \mathrm{T}_{\max }(\mathrm{h})$ and $\mathrm{AUC}_{0-\infty}(\mathrm{ng} / \mathrm{mL} * \mathrm{~h})$. Relative bioavailability (RBA) of the $\mathrm{NCH} 1$ and $\mathrm{NCH} 2$ was calculated as follow:

$$
\begin{aligned}
R B A(\%)= & 100 \\
& * A U C(0-\infty) \text { of NCHs } / A U C(0-\infty) \text { ofTG }
\end{aligned}
$$

\section{Statistical Analysis}

Data were statistically analyzed by the ANOVA (analysis of variance) using SPSS-16 software.

Statistical analysis was performed, represented as mean $\pm \mathrm{SD}(\mathrm{n}=5)$. A $\mathrm{p}$-value $<0.05$ was considered significant statistically. ${ }^{13}$

\section{Results}

\section{Characterization of Thiolated Chitosan (TCH)}

\section{FT-IR Spectroscopy}

Chitosan was successfully modified in the form of $\mathrm{TCH}$. FT-IR spectra of TCH indicated successful modification as indicated by the presence of a peak at $1646 \mathrm{~cm}^{-1}$ showing the presence of stretching amide $(-\mathrm{N}-\mathrm{H}$ stretch) whereas peaks at $1246 \mathrm{~cm}^{-1}$ and $2495 \mathrm{~cm}^{-1}$ confirmed the presence of thiol groups (C-SH stretch) as represented in Figure 3.

\section{Quantification of Thiol Content}

The amount of thiol moieties has been quantified from the standard curve of TGA solutions prepared in water in 


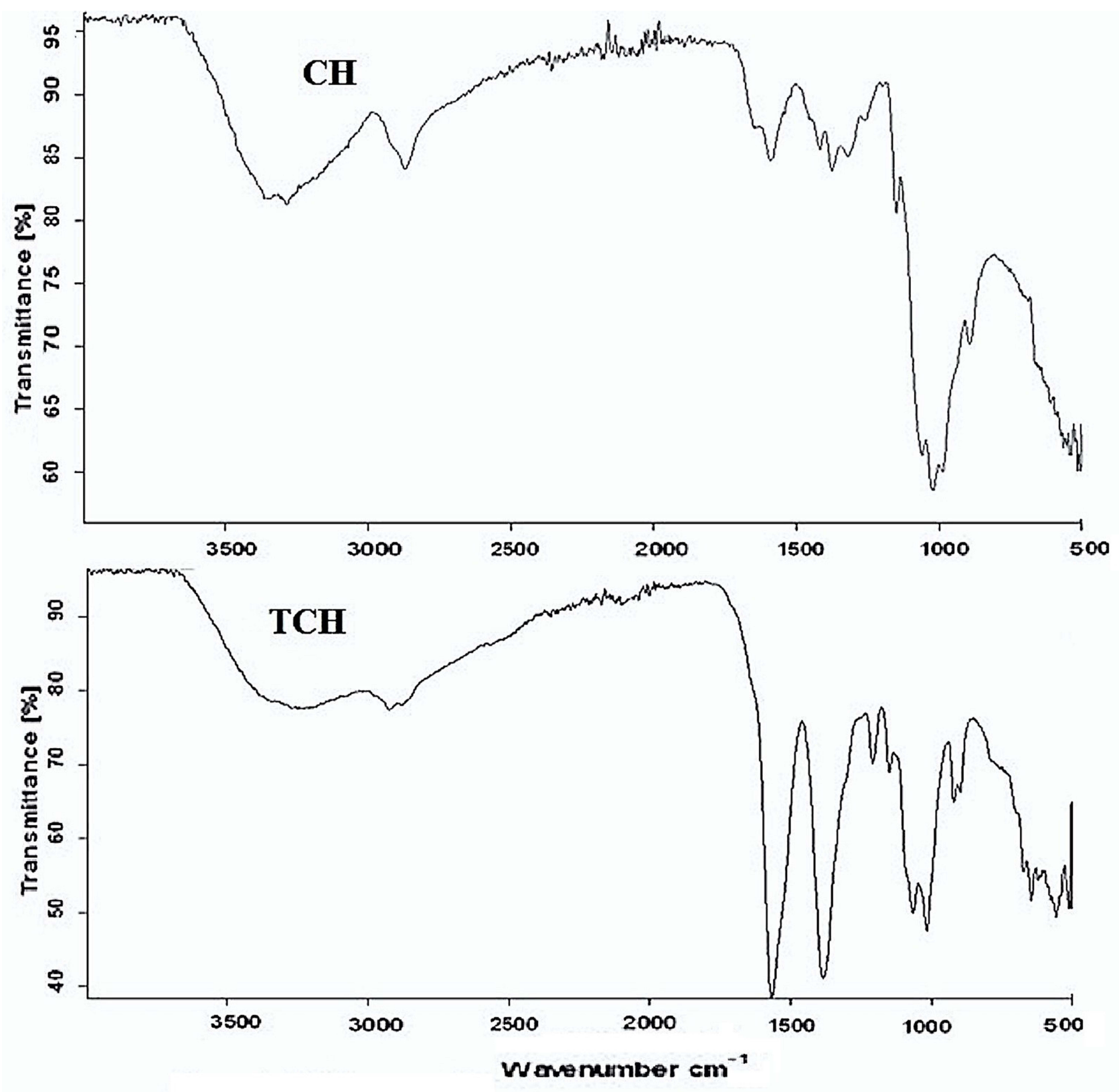

Figure 3 FT-IR spectra of chitosan and thiolated chitosan.

Abbreviations: FT-IR, Fourier transform infra-red spectroscopy; $\mathrm{CH}$, chitosan; $\mathrm{TCH}$, thiolated chitosan.

a concentration range of 0.25 to 2 millimoles (mM). The modified $\mathrm{CH}(\mathrm{TCH})$ displayed $465 \mathrm{mM}$ of thiol groups present per gram of polymer. ${ }^{11}$

\section{Characterization of $\mathrm{CH}-\mathrm{NPs}$ and TC-NPs}

Findings of basic characterization done on chitosan and thiolated chitosan nanoparticles, CH-NPs and TC-NPs are represented in Table 3. Entrapment efficiency (EE) was determined by HPLC by quantifying the free drug concentration in the supernatant solution obtained after centrifugation. FT-IR spectrum and DSC thermogram of TC-NPs have been demonstrated in Figures 4 and 5, respectively.

\section{Characterization of Hydrogel}

\section{Formulations}

\section{Porosity}

The porosity of all nine formulations was determined by the solvent replacement method. The results presented in Figure 6 indicated that porosity increased by increasing both the monomer (AA) and polymer's $(\mathrm{CH})$ concentrations. 


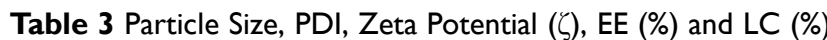
of $\mathrm{CH}-\mathrm{NPs}$ and TC-NPs

\begin{tabular}{|l|c|l|l|l|l|}
\hline Formulations & $\begin{array}{c}\text { Particle } \\
\text { Size } \\
\text { (nm) }\end{array}$ & PDI & $\begin{array}{l}\text { Zeta } \\
\text { Potential } \\
\text { (mv) }\end{array}$ & $\begin{array}{c}\text { EE } \\
\text { (\%) }\end{array}$ & $\begin{array}{l}\text { LC } \\
\text { (\%) }\end{array}$ \\
\hline CH-NPs & 147.3 & 0.364 & 22.6 & 84.1 & 42.05 \\
TC-NPs & 190.3 & 0.375 & 16.1 & 91.3 & 38.0 \\
\hline
\end{tabular}

Abbreviations: PDI, polydispersity index; EE, entrapment efficiency; LC, loading capacity; CH-NPs, chitosan nanoparticles; TC-NPs, thiolated chitosan nanoparticles.

It possibly occurred due to an increase in viscosity by increasing $\mathrm{AA}$ and $\mathrm{CH}$ content that resulted in the formation of porous interlinked channels. Whereas, it decreased by increasing MBAA (crosslinker) concentration, due to enhanced entanglements between $\mathrm{AA}$ and $\mathrm{CH}$, increasing crosslinking density which ultimately reduced pore formation and hence decreased porosity.

\section{Sol-Gel Fraction}

Gel contents and sol contents were determined for all formulations as presented in Figure 7 below. Sol fraction decreased but gel fraction increased by increasing $\mathrm{CH}$, AA, and MBAA concentrations. An increase in gel fraction can be attributed to the fact that by increasing polymer $(\mathrm{CH})$, monomer (AA), and cross-linker (MBAA) contents, improved crosslinking occurs bringing an increase in gel strength. Similar findings have been reported by Ranjha

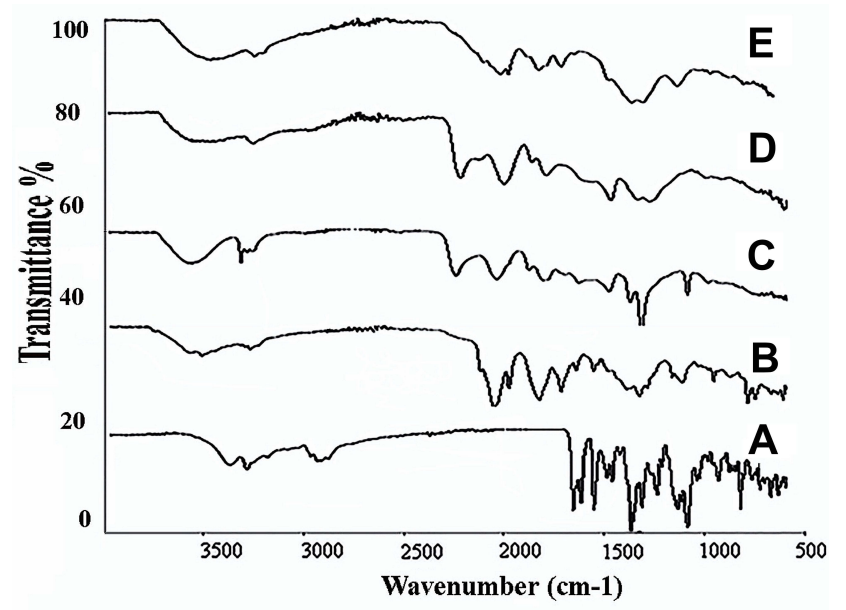

Figure 4 Fourier transform infra-red spectroscopy data. (A) TG, (B) TC-NPs, (C) DLH, (D) NCHI, and (E) NCH2. Abbreviations: FT-IR, Fourier transform infra-red spectroscopy; TG, ticagrelor; TC-NPs, thiolated chitosan nanoparticles.

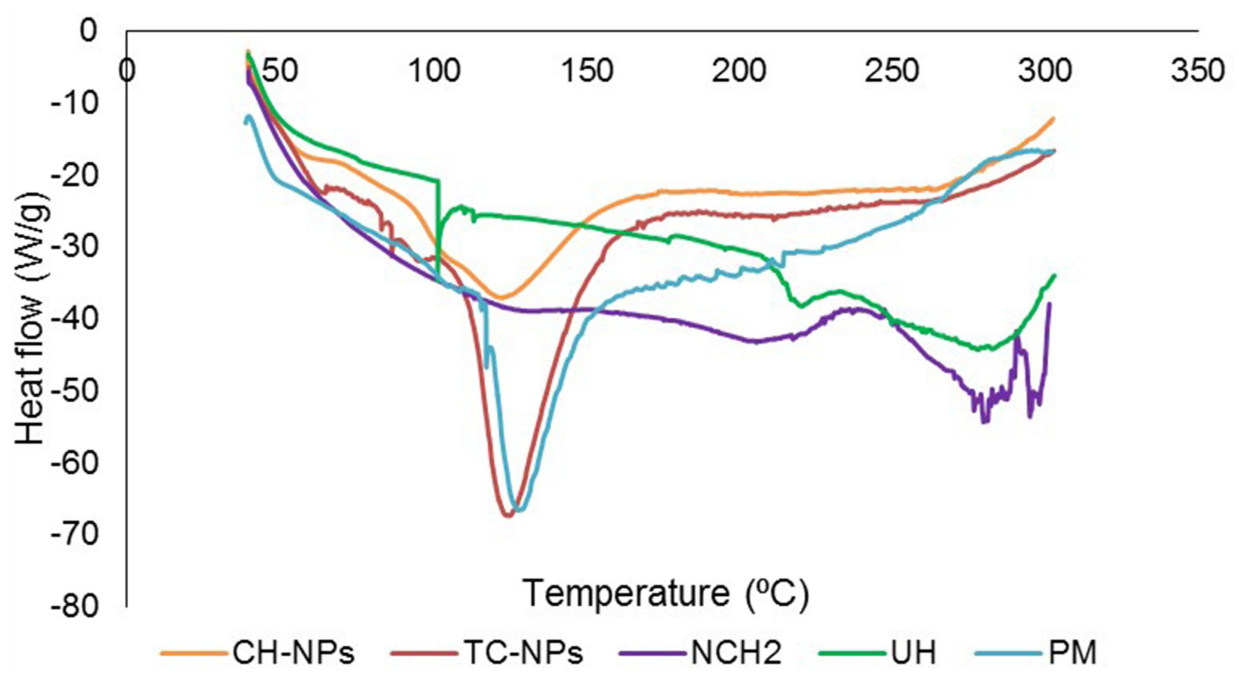

Figure 5 Differential scanning calorimetry (DSC) thermograms of tTicagrelor (TG); chitosan nanoparticles (CH-NPs); thiolated chitosan nanoparticles (TC-NPs); nanocomposite hydrogel formulation ( $\mathrm{NCH} 2)$; physical mixture (PM); and unloaded hydrogels $(\mathrm{UH})$. 


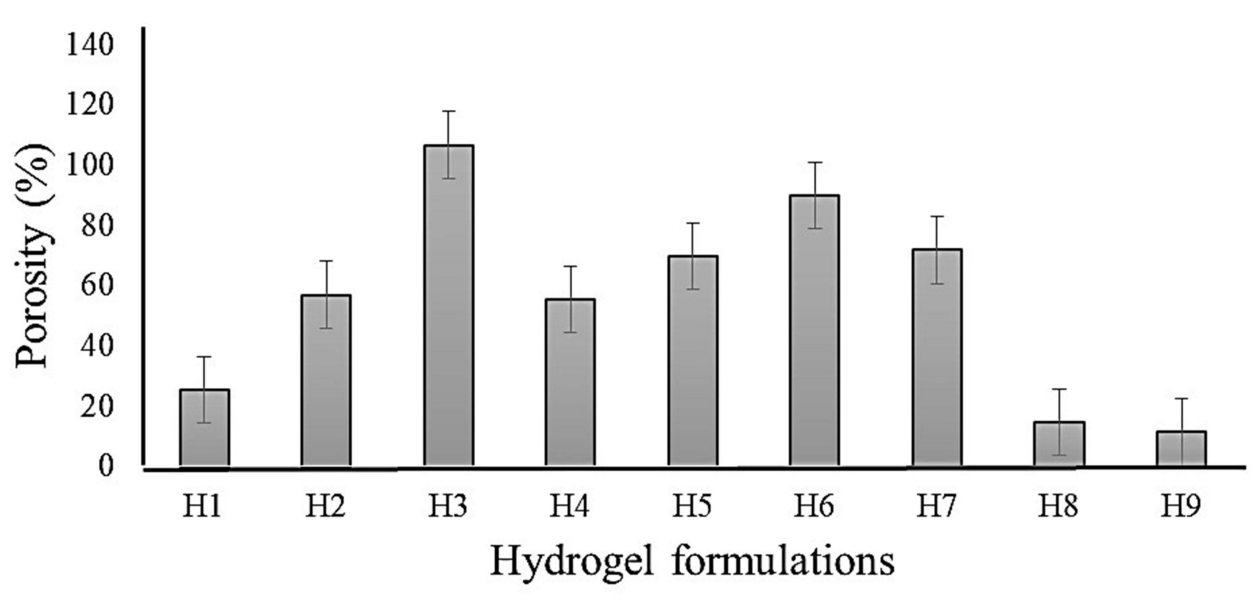

Figure 6 Porosity determination of $\mathrm{CH}$-co-AA formulations $(\mathrm{HI}-\mathrm{H} 9)$.

Abbreviations: $\mathrm{CH}-\mathrm{co}-\mathrm{AA}$, chitosan-co-acrylic acid; $\mathrm{HI}-\mathrm{H}$ 9, various hydrogel formulations.

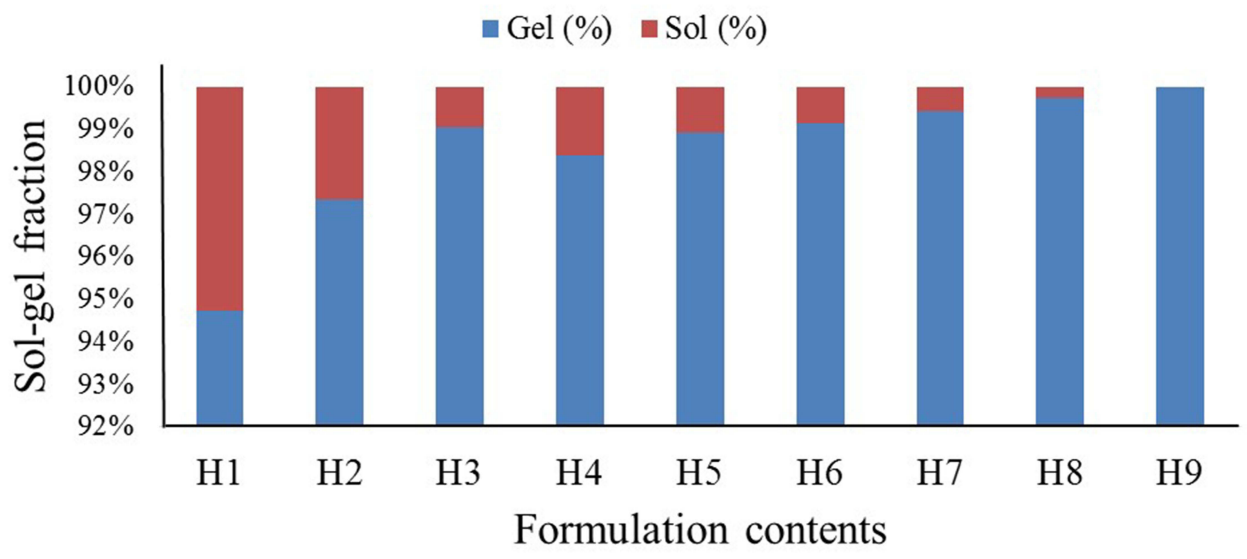

Figure 7 Sol-gel fraction of chitosan-co-acrylic acid formulations.

Abbreviation: $\mathrm{CH}-\mathrm{co}-\mathrm{AA}$, chitosan-co-acrylic acid.

et al indicating a decrease in porosity but an increase in gel content upon increasing MBAA content.

\section{Swelling Studies}

Effect of $\mathrm{pH}$ on Swelling Behavior

A pH-dependent swelling behavior was observed by all hydrogel formulations ( $\mathrm{H} 1-\mathrm{H} 9)$, upon dynamic and equilibrium swelling studies performed for 72 hours (-figure 10). USP buffer solutions of various $\mathrm{pH} 1.2,4.5,6.8$, and 7.4 were employed to observe swelling behavior towards varying physiological $\mathrm{pH}$. Dynamic swelling (swelling ratio, q) was studied at $\mathrm{pH} 1.2$ and 7.4. For equilibrium swelling studies additional $\mathrm{pH}$ media were selected $(\mathrm{pH}$ 4.5 and 6.8).

Effect of $\mathrm{CH}$ Concentration Upon Swelling Behavior $\mathrm{CH}$ concentration was progressively increased in the first three formulations $(\mathrm{H} 1-\mathrm{H} 3)$ from $0.3 \% \mathrm{w} / \mathrm{w}$ to $0.7 \% \mathrm{w} / \mathrm{w}$. An increased swelling behavior was observed by increasing $\mathrm{CH}$ concentration at low acidic $\mathrm{pH}$ (1.2), but not significantly, ie from $51.2 \%$ to $58.8 \%$ ES. It was due to the ionization of $-\mathrm{NH}_{2}$ groups of $\mathrm{CH}$ in the network.

\section{Effect of AA Concentration Upon Swelling Behavior}

Upon incrementing AA content, improved swelling behavior was observed in the alkaline medium as compared to that displayed in the acidic medium. Both swelling ratio (q) and \%ES increased slowly by increasing monomer (AA) content as displayed in Figures 9B and $10 \mathrm{~B}$.

Effect of MBAA Concentration Upon Swelling Behavior Three formulations (H7-H9) were fabricated comprising varied cross-linker (MBAA) ratios. An inverse 


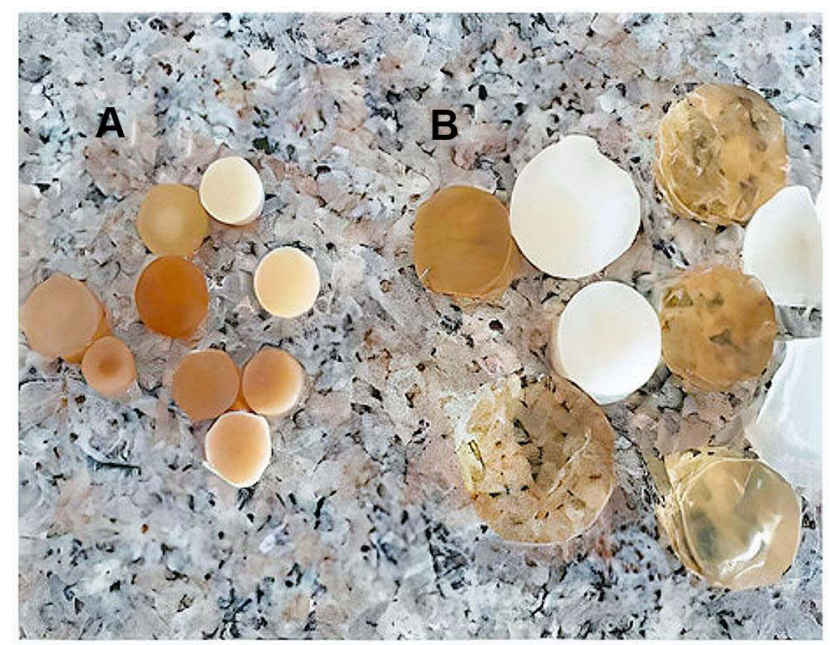

Figure 8 Swelling behavior of chitosan-co-acrylic acid hydrogels. (A) pH I.2 and (B) At pH 7.4 Abbreviation: CH-co-AA, chitosan-co-acrylic acid.
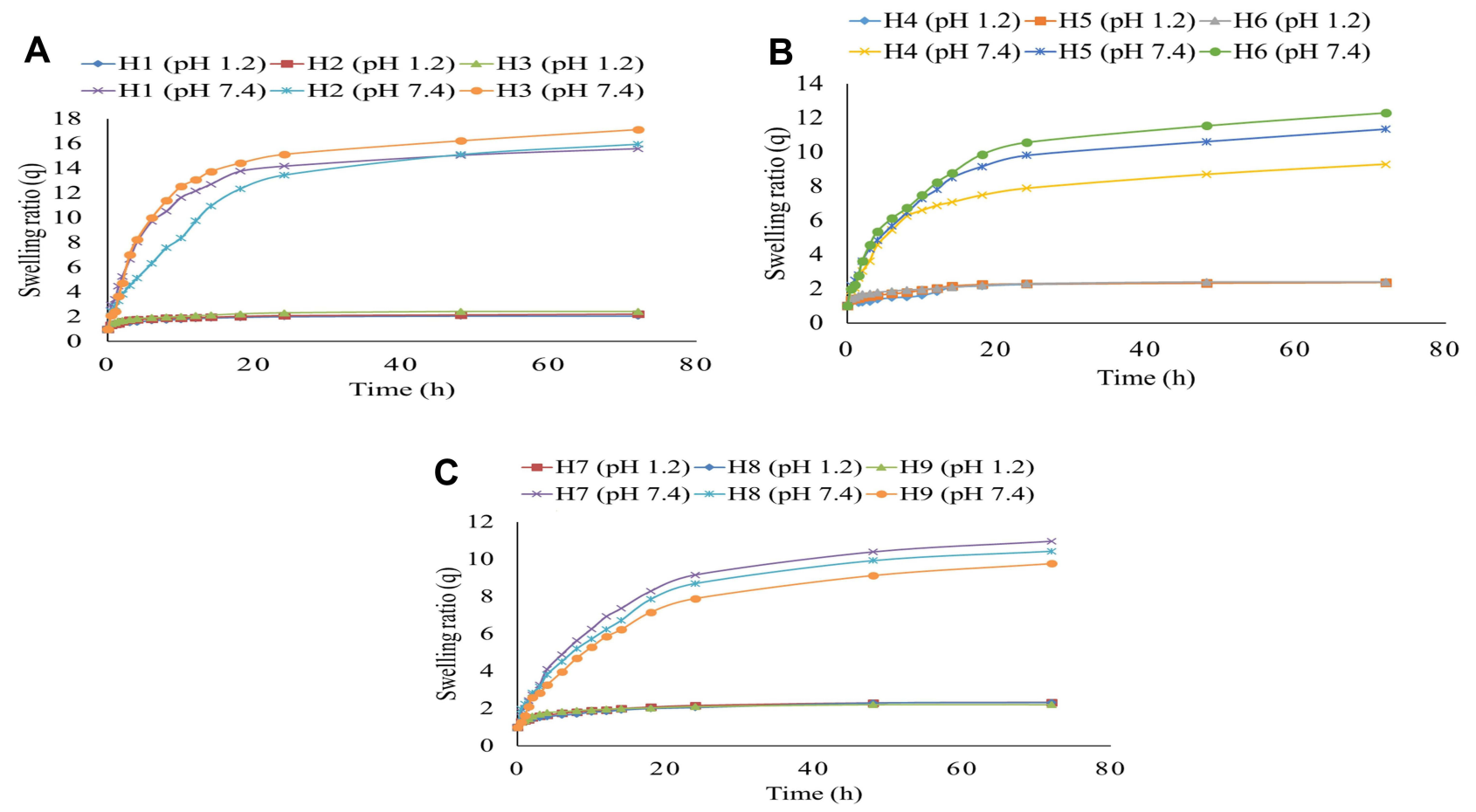

Figure 9 Swelling ratios (q) of $\mathrm{CH}$-co-AA formulations. (A) varying $\mathrm{CH}$ concentration $(\mathrm{HI}-\mathrm{H} 3)$ (B) varying $\mathrm{AA}$ concentration (H4-H6), and (C) varying MBAA concentration ( $\mathrm{H7}-\mathrm{H} 9)$. Abbreviations: $\mathrm{CH}-\mathrm{co}-\mathrm{AA}$, chitosan-co-acrylic acid; $\mathrm{HI}-\mathrm{H}$, various hydrogel formulations; $\mathrm{CH}$, chitosan; $\mathrm{AA}$, acrylic acid; MBAA, methylene bis acrylic acid.

relationship between MBAA concentration and swelling efficiency was observed ie by increasing crosslinker content, swelling ratio, $\mathrm{q}$, and \%ES was decreased as shown in Figures 9C and 10C. Higher crosslinker (MBAA) content increases the cross-linking density of the polymeric network. Increased cross-linking tightens the structure of hydrogel that impedes diffusion and hence decreases the swelling potential.

\section{Drug Loading}

The drug loading capacity of various formulations of $\mathrm{CH}-$ co-AA is presented in Table 4. 


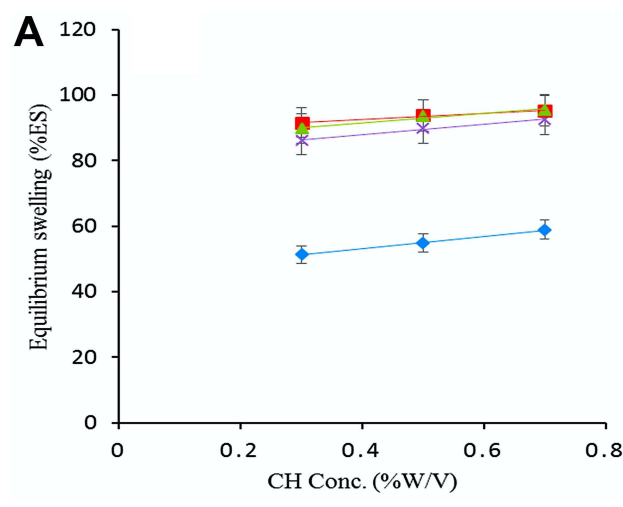

- $\mathrm{pH} 1.2$

$\times \mathrm{pH} 4.5$

- $\mathrm{pH} 6.8$

$\triangle \mathrm{pH} 7.4$

— Linear (pH 1.2)

_ Linear ( $\mathrm{pH} 4.5$ )

_ Linear ( $\mathrm{pH} 6.8$ )

Linear $(\mathrm{pH} 7.4)$
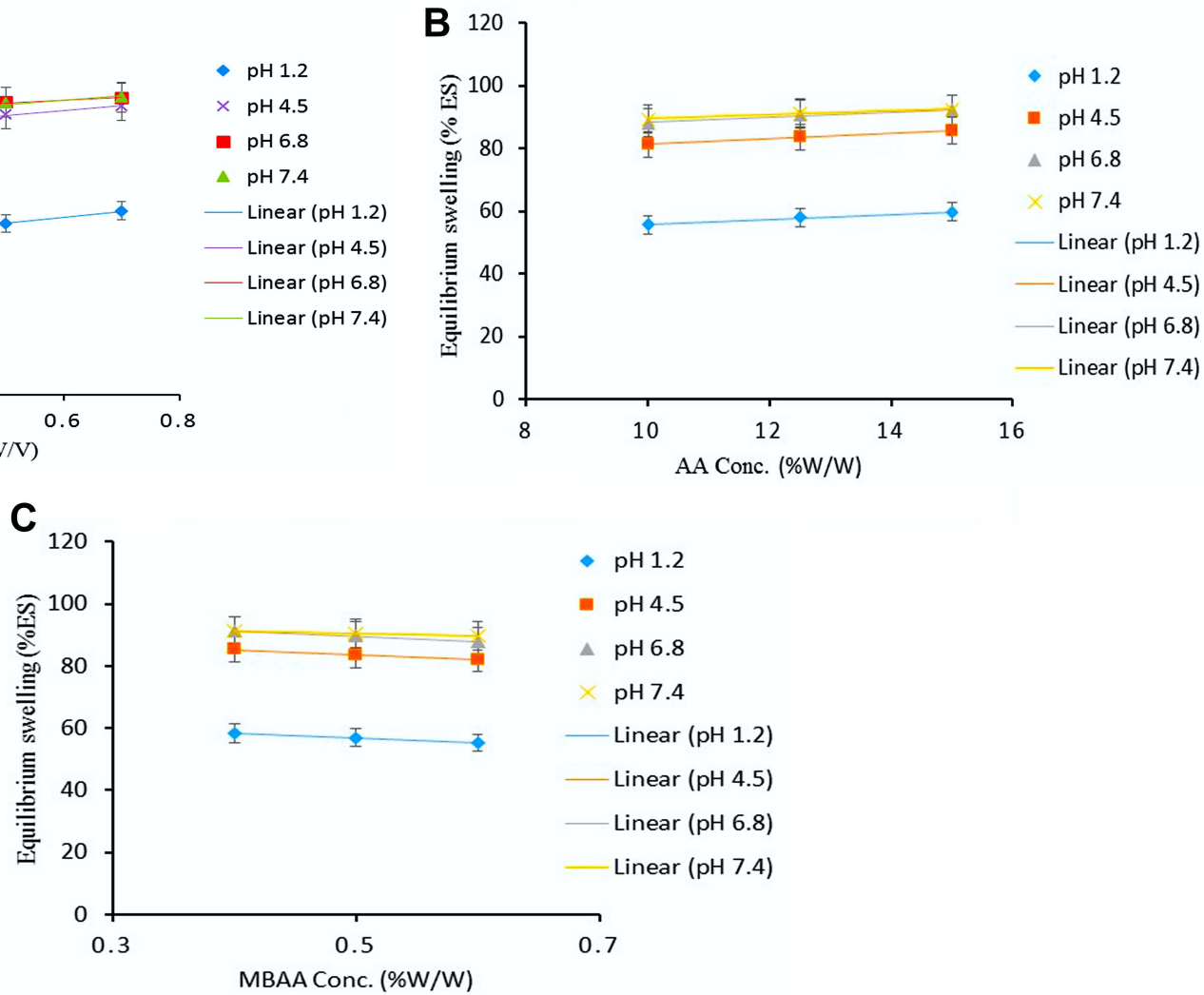

Figure 10 Percent ES of chitosan-co-acrylic acid formulations. (A) Varying $\mathrm{CH}$ concentration (HI-H3), (B) varying AA concentration (H4-H6), and (C) varying MBAA concentration $(\mathrm{H} 7-\mathrm{H}$ 9). Abbreviations: $\mathrm{HI}-\mathrm{H}$ 9, various hydrogel formulations; $\mathrm{CH}$, chitosan; $\mathrm{AA}$, acrylic acid; MBAA, methylene bis acrylic acid.

\section{FT-IR Spectroscopy}

To assess structural modifications and confirm the presence of specific functional groups in cross-linked hydrogels, NCHs along with pure TG and TG-loaded TC-NPs

Table 4 Drug Loading Capacity in All Formulations of $\mathrm{CH}$-coAA Hydrogels

\begin{tabular}{|l|c|c|}
\hline $\begin{array}{l}\text { Formulation } \\
\text { Code }\end{array}$ & $\begin{array}{c}\text { By Weight Method } \\
\text { (mg/g Disc) }\end{array}$ & $\begin{array}{c}\text { By Extraction } \\
\text { Method (mg/g Disc) }\end{array}$ \\
\hline $\mathrm{HI}$ & 482.3 & 495.1 \\
$\mathrm{H} 2$ & 521.0 & 527.8 \\
$\mathrm{H} 3$ & 593.2 & 601.2 \\
$\mathrm{H} 4$ & 381.7 & 389.0 \\
$\mathrm{H} 5$ & 587.2 & 593.4 \\
$\mathrm{H} 6$ & 611.3 & 615.7 \\
$\mathrm{H} 7$ & 583.0 & 587.1 \\
$\mathrm{H} 8$ & 497.1 & 499.0 \\
$\mathrm{H} 9$ & 356.4 & 362.9 \\
$\mathrm{NCHI}$ & 543.1 & 547.2 \\
$\mathrm{NCH} 2$ & 583.3 & 585.6 \\
\hline
\end{tabular}

Abbreviations: $\mathrm{CH}$-co-AA, chitosan-co-acrylic acid; $\mathrm{HI}$ to $\mathrm{H}$, hydrogel formulations; $\mathrm{NCHI}$ and $\mathrm{NCH}$, nanocomposite hydrogel formulations. were subjected to FT-IR spectroscopy. The FT-IR spectrum obtained from pure TG displayed major peaks at $3288,2930,2853,1604,1558,1455,1274,1210,1110$ and $1091 \mathrm{~cm}^{-1}$. $-\mathrm{NH}$ and $-\mathrm{OH}$ stretch were confirmed by the presence of absorption bands at $3288 \mathrm{~cm}^{-1}$, while specific peaks at 1604 and $1558 \mathrm{~cm}^{-1}$ displayed the presence of $-\mathrm{NH}$ stretch (Figure 4).

\section{Morphological Analysis (SEM)}

Both unloaded hydrogels and drug-loaded nanocomposites ( $\mathrm{NCH} 1$ and $\mathrm{NCH} 2$ ) were subjected to scanning electron microscopy (SEM) to study the surface morphology of formulated hydrogel networks. SEM micrographs demonstrated a rough surface morphology of unloaded and loaded hydrogels. Moreover, surface morphology revealed porous surfaces comprised of voids and interpenetrating channels in all hydrogel formulations possibly due to the presence of ionic or hydrophilic groups throughout the hydrogel framework. SEM micrographs illustrated evenly embedded nanoparticles throughout the composite network. SEM images of $\mathrm{NCH}$ formulations have been displayed in Figure 11. 


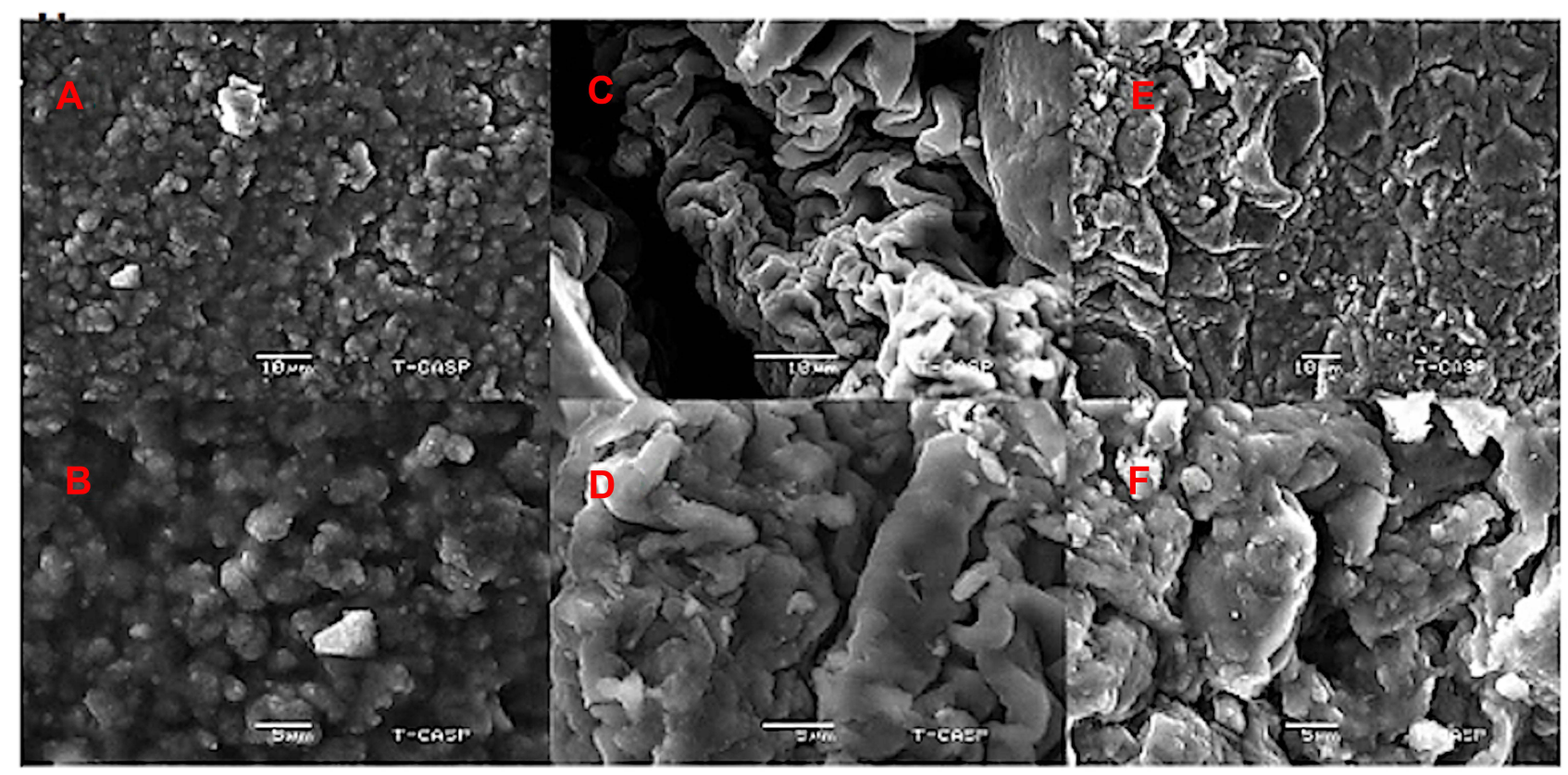

Figure I I Scanning electron microscopy images. (A and B) unloaded hydrogels, (C and $\mathbf{D}) \mathrm{NCHI}$, (E and $\mathbf{F}) \mathrm{NCH} 2$. Abbreviations: $\mathrm{NCHI}$ and $\mathrm{NCH} 2$, nNanocomposite hydrogel formulations.

\section{DSC Analysis}

Thermal analysis was performed to investigate the crystalline nature of pure TG, unloaded hydrogel matrix, thiolated chitosan nanoparticles (TC-NPs), physical mixture, and nanocomposite hydrogel $(\mathrm{NCH} 2)$ as shown in Figure 5. In the physical mixture, TG's endothermic peak along with other small peaks was observed. In the case of TG-loaded nanoparticles (TC-NPs), a broadened and early endothermic peak was observed at $124.9{ }^{\circ} \mathrm{C}$ indicating possible interactions among $\mathrm{CH}$ and $\mathrm{TG}$ along with the reduction in the crystalline nature of enclosed TG.

\section{PXRD Analysis}

X-ray diffraction analysis was performed to evaluate crystallinity or amorphicity of pure TG, TG loaded copolymers (DLH), and nanocomposite hydrogels (NCH1 and $\mathrm{NCH} 2$ ). Each diffractogram exhibited a distinctive pattern and peak intensity. XRD of pure TG displayed intense peaks at $5.35^{\circ}, 6.65^{\circ}, 13.35^{\circ}, 19^{\circ}, 22.5^{\circ}$, and $24.2^{\circ}$, conforming to its crystalline nature. Moreover, $\mathrm{NCH} 1$ and $\mathrm{NCH} 2$ displayed less intense peaks as compared to that of pure TG and $\mathrm{CH}-\mathrm{NPs}$. NCH1 displayed major peaks at $5.4^{\circ}, 5.8^{\circ}, 8.35^{\circ}, 11.35^{\circ}$, and $15.1^{\circ}$, whereas, $\mathrm{NCH} 2$ displayed major peaks at $5.3^{\circ}, 6.05^{\circ}, 16.75^{\circ}$, $17.45^{\circ}, 19.35^{\circ}, 20.3^{\circ}, 21$, and $21.3^{\circ}$ (Figure 12).

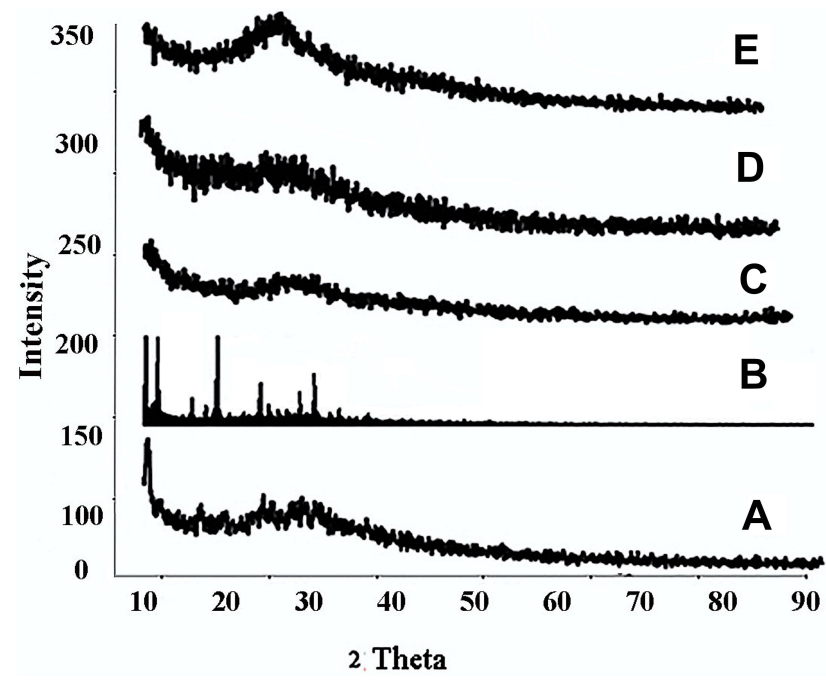

Figure 12 Powder X-ray diffraction patterns. (A) TC-NPs, (B) TG, (C) DLH, (D) $\mathrm{NCHI}$, and (E) NCH2. PXRD, Powder X-ray diffraction.

Abbreviations: TC-NPs, thiolated chitosan nanoparticles; TG, ticagrelor; DLH, drug loaded hydrogels; $\mathrm{NCHI}$ and $\mathrm{NCH}$, nanocomposite hydrogel formulations.

\section{In Vitro Release Studies}

Release kinetics were studied for 2 hours in acidic buffer $(\mathrm{pH} 1.2)$ and then for a further 22 hours in USP phosphate buffer ( $\mathrm{pH}$ 7.4). Release profiles of formulations having varying concentrations of $\mathrm{CH}, \mathrm{AA}$ and MBAA are presented in Figure 13. All formulations demonstrated a $\mathrm{pH}-$ 

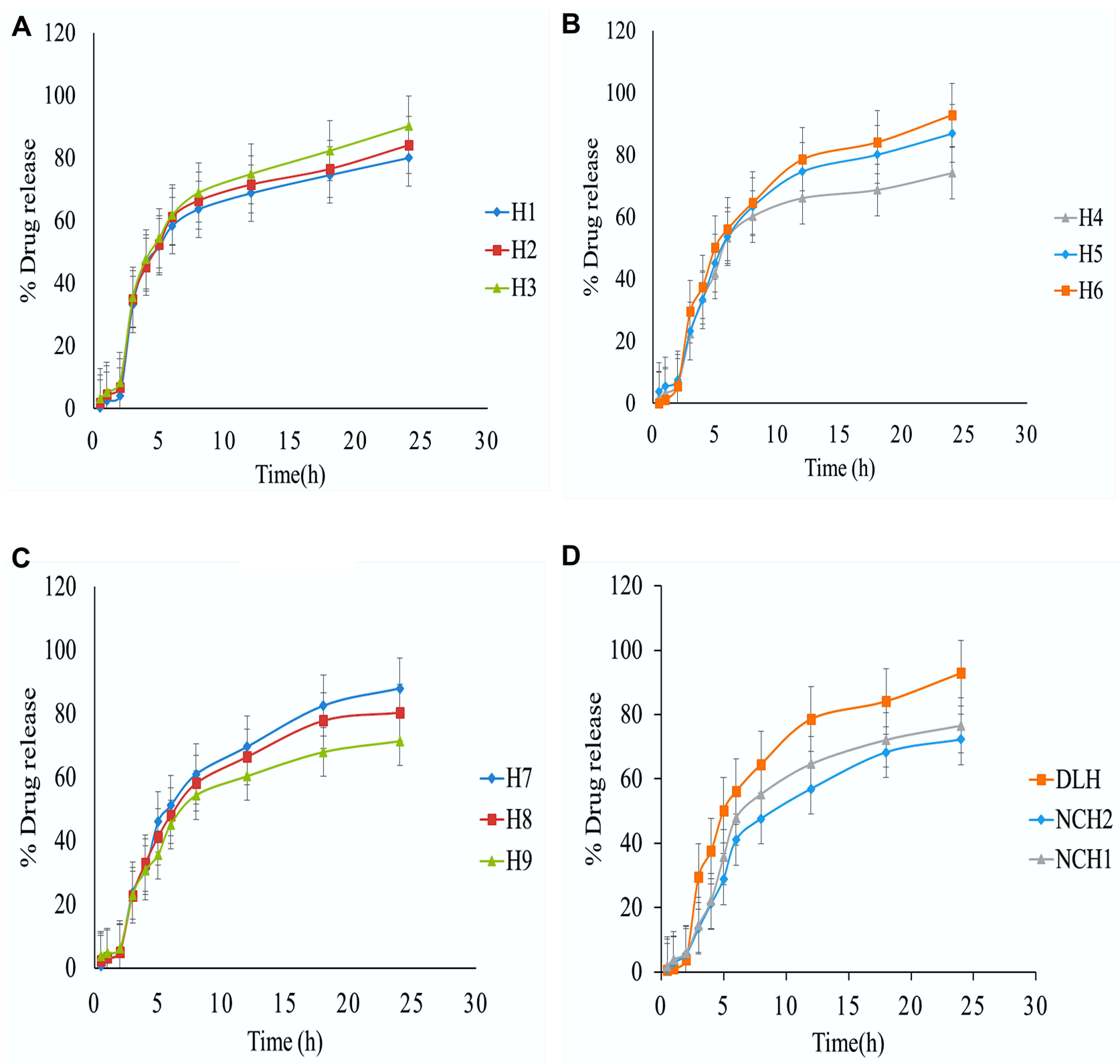

Figure 13 In vitro release profiles of chitosan-co-acrylic acid formulations. (A) Varying CH ratio, (B) varying AA ratio, (C) varying MBAA ratio, and (D) comparative in vitro profiles of $\mathrm{DLH}, \mathrm{NCHI}$ and $\mathrm{NCH} 2$

Abbreviations: $\mathrm{CH}$-co-AA, chitosan-co-acrylic acid; $\mathrm{HI}-\mathrm{H}$ 9, various hydrogel formulations; $\mathrm{CH}$, chitosan; AA, acrylic acid; MBAA, methylene bis acrylic acid; DLH, drug loaded hydrogels; $\mathrm{NCHI}$ and $\mathrm{NCH} 2$, nanocomposite hydrogel formulations.

dependent release behavior. The cumulative \% drug release $(\% \mathrm{DR})$ observed from all formulations was less in $\mathrm{pH} 1.2$ as compared to that in $\mathrm{pH}$ 7.4. All formulations displayed a \%DR of less than $10 \%$ in the first two hours in an acidic medium. This behavior was also seen in the case of swelling studies. The rate of drug release is dependent on the EE of the hydrogel matrix, as more drug release was exhibited by those formulations having a high EE.

\section{Evaluation of Drug Release Kinetics}

Drug release data of TG was evaluated for all formulations by applying various models such as zero order, first order, Higuchi, Korsmeyer-Peppas, and HixsonCrowell, using DD solver software. Results of kinetic assessment are presented in Table 5, along with values of the regression coefficient $\left(\mathrm{R}^{2}\right)$ and rate constants $(\mathrm{k})$ for all models. 
Table 5 Correlation Coefficients (R2) and Release Rate Constants of $\mathrm{CH}$-co-AA and NCH Formulations

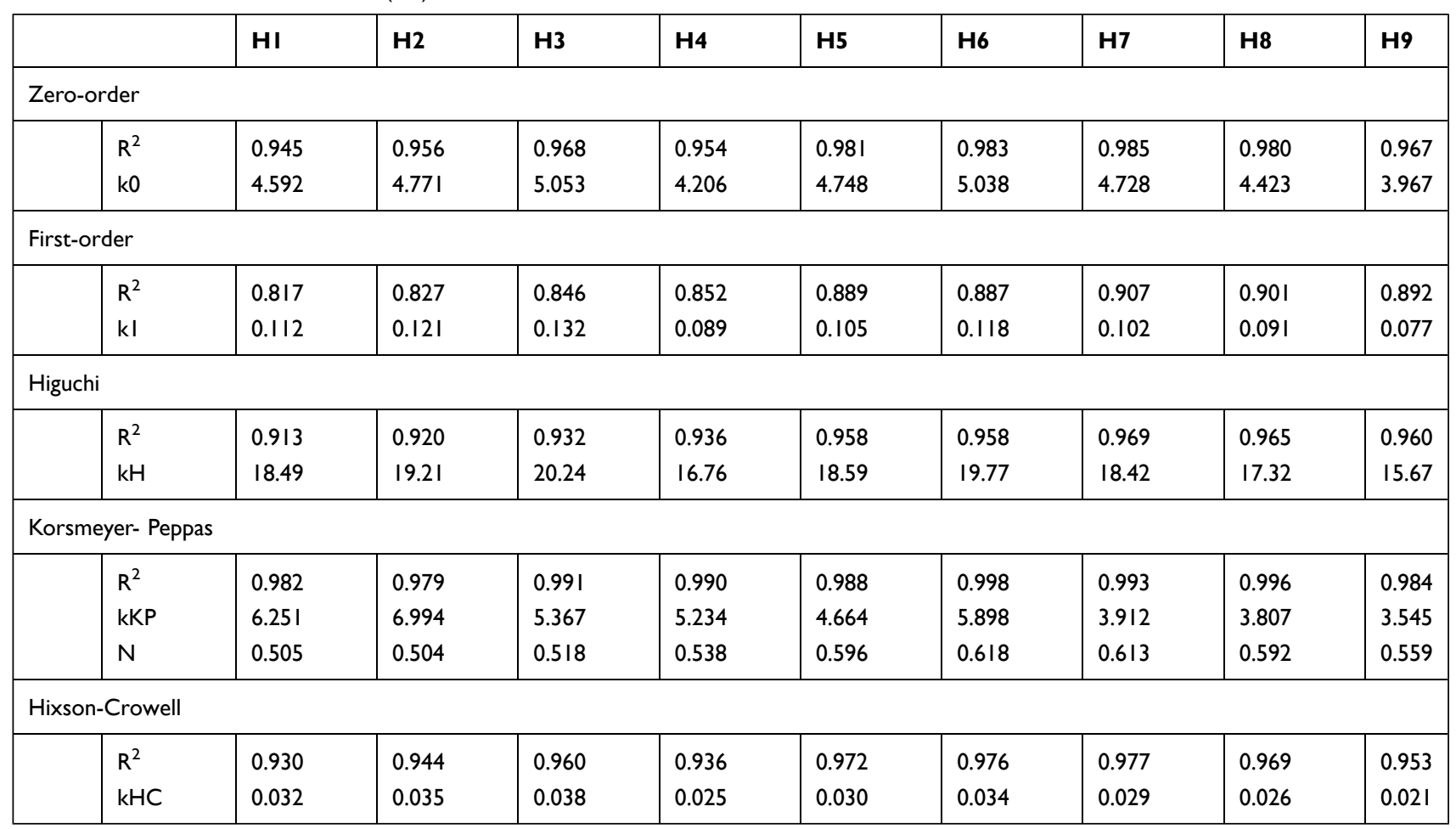

Abbreviations: $\mathrm{CH}$-co-AA, chitosan-co-acrylic acid; $\mathrm{NCH}$, nanocomposite hydrogels; $\mathrm{HI}$ to $\mathrm{H}$, hydrogel formulations.

\section{Acute Oral Toxicity Study}

At an administered dose of $5000 \mathrm{mg} / \mathrm{kg}$ body weight, no signs of toxicity were observed in treatment groups G2 and G3 for 14 days. Likewise, no mortality was observed due to acute oral toxicity in both treatment groups similar to the control group, G1. Treatment groups displayed normal behaviors like that of the control group. No signs of diarrhea, vomiting, edema, fatigue, weight loss, or eye lacrimation were observed. Results of histopathological inspection of vital organs are presented in Figure 14.

\section{In Vivo Bioavailability Study}

Pharmacokinetic parameters were evaluated for nanocomposite formulations, $\mathrm{NCH} 1$ and $\mathrm{NCH} 2$, and were compared with that of pure TG after administering equivalent oral dose $(10 \mathrm{mg} / \mathrm{kg})$ to rabbits. Plasma level time curves were drawn to calculate pharmacokinetic parameters such as maximum systemic concentration $\left(\mathrm{C}_{\max }\right)$, time to reach maximum systemic concentration $\left(\mathrm{t}_{\max }\right)$, area under the curve $\left(\mathrm{AUC}_{0-\infty}\right)$, and half-life $\left(\mathrm{t}_{1 / 2}\right)$ and are presented in Table 6. Both $\mathrm{C}_{\max }$ and $\mathrm{T}_{\max }$ were observed to be higher as compared to that of TG (Figure 15).

\section{Discussion}

Cardiovascular diseases such as myocardial infarction, cardiac failure, and stroke are emerging as massive challenges in recent years. TG is an effective drug employed for treating stroke and myocardial infarction in patients with acute coronary syndrome, but its short duration of action and low oral bioavailability hinder its effectiveness. To overcome such issues related to TG's insufficient solubility and permeability, an innovative $\mathrm{NCH}$ formulation was fabricated incorporating TCH nanoparticles utilizing TCH as a mucoadhesive polymer. NCHs amalgamate the advantages of both nanoparticles and hydrogel matrices that result in their enhanced biological and mechanical characteristics holding promising applications in the biomedical field.

The initial step was a modification of polymer and preparation of nanoparticles (NPs) containing TG. Chitosan (CH) was successfully modified by thiolation as confirmed by FT-IR spectroscopy (Figure 3) and the presence of thiol content (465 $\mathrm{mmol} / \mathrm{g}$ ). Thiolation of $\mathrm{CH}$ will promote mucoadhesion and being an absorption enhancer will result in efficient absorption due to disulfide bond formation between its thiol groups and cysteine-rich glycoproteins of the mucus membrane. This 


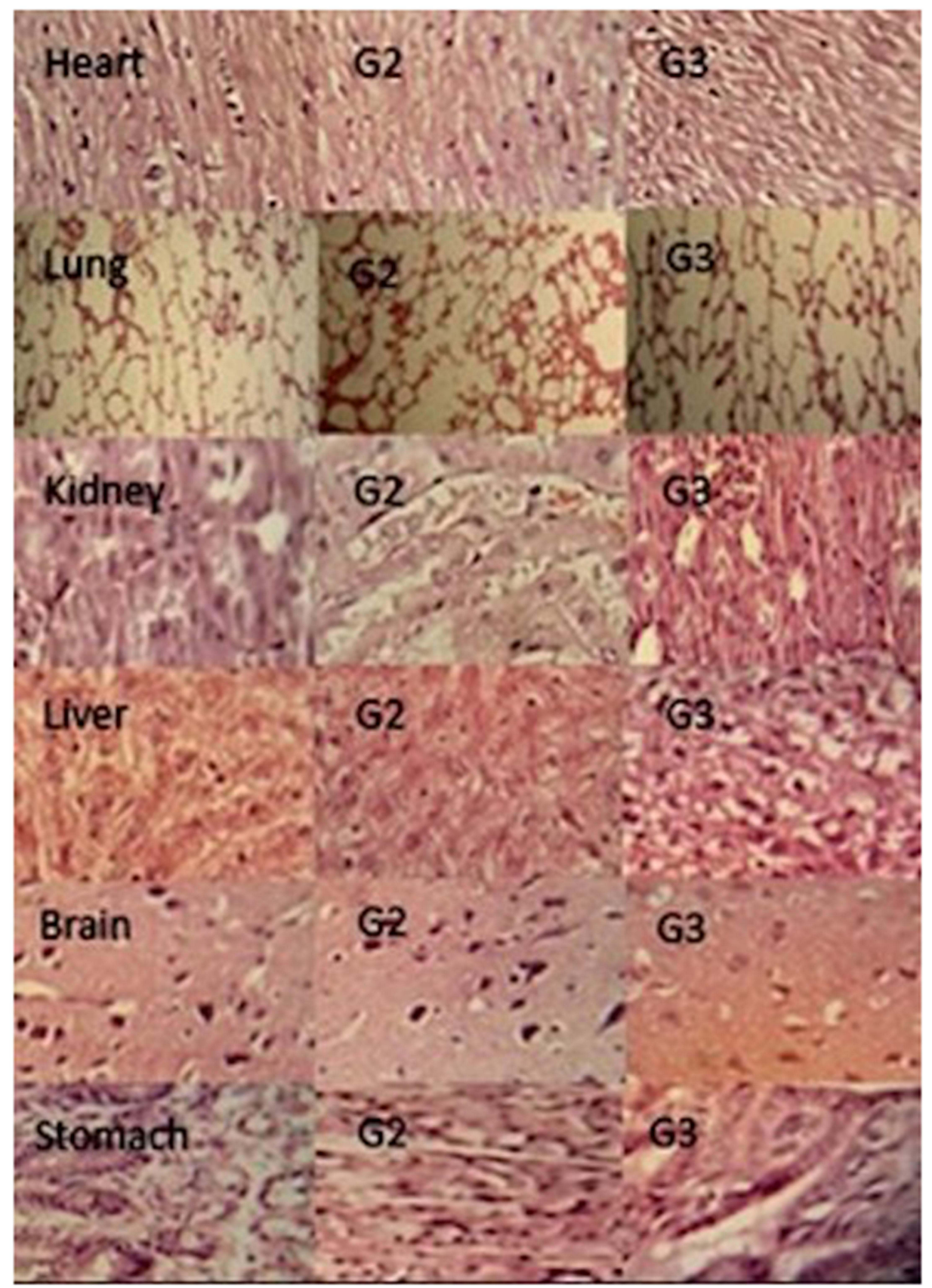

Figure 14 Histopathological observations of tissues from organs of groups GI, G2, and G3 including heart, lungs, kidney, liver, brain, and stomach as observed in an acute oral toxicity study. Abbreviations: GI, group I; G2, group 2; G3, group 3.

linkage is stronger as compared to non-covalent interactions between the anionic mucus layer and cationic $\mathrm{CH}$. Likewise, CH-NPs and TC-NPs were favorably fabricated by ionic gelation and were characterized efficiently. CH-NPs and TCNPs displayed nano-sized particulate carriers having a size less than $150 \mathrm{~nm}$ and $200 \mathrm{~nm}$, respectively, along with entrapment efficiency (EE) of $>80 \%$ and $>90 \%$, respectively. The ratio of polymer $(\mathrm{CH})$ to crosslinker (TPP) and $\mathrm{TG}$ concentration had a great impact on NP formation, particle size, zeta potential, and loading capacity. Preliminary studies demonstrated a reduction in particle size by increasing polymer concentration $(\mathrm{CH})$ with respect to the drug (TG) and TPP. Zeta potential that reflects the repulsion or attraction among adjoining particles, demonstrated an increased value by increasing $\mathrm{CH}$ concentration, possibly due to neutralization of charges between negatively charged TPP and positive 
Table 6 Pharmacokinetic Parameters After a Single Oral Dose of Pure TG, NCHI, and NCH2 in Rabbits. Data Expressed as Mean \pm $\operatorname{SD}(n=5)(p<0.05)$

\begin{tabular}{|l|l|l|l|l|l|}
\hline Samples & Cmax $(\mathbf{n g} / \mathbf{m L})$ & $\mathbf{T m a x}(\mathbf{h})$ & $\mathbf{t}_{1 / 2}(\mathbf{h})$ & AUCo- $(\mathbf{n g} / \mathbf{m L}$ *h) & RBA (\%) Against Pure TG \\
\hline Pure TG & 88.98 & 2 & 7.737 & 1034.72 & \\
$\mathrm{NCHI}$ & 584.33 & 6 & 11.60 & 9789.42 & 946.09 \\
$\mathrm{NCH} 2$ & 642.1 & 6 & 13.09 & $11,620.74$ & 1123.08 \\
\hline
\end{tabular}

Abbreviations: TG, ticagrelor; $\mathrm{NCHI}$ and $\mathrm{NCH} 2$, nanocomposite hydrogel formulations; Cmax, maximum plasma concentration; Tmax, time for maximum concentration; $t_{1 / 2}$, half-life; AUC, area under the curve; RBA, relative bioavailability.

amine groups of $\mathrm{CH}$. Previous studies have also reported that NPs, having a size of $<200 \mathrm{~nm}$, display efficient stability, cellular uptake for site-specific delivery, and prolonged circulation time. $^{19}$

Various hydrogel formulations (H1-H9), having varying polymer $(\mathrm{CH})$, monomer $(\mathrm{AA})$ and crosslinker (MBAA) contents were effectively developed by free radical polymerization technique as presented in Table 2. H6 was selected as an optimized formulation for loading $\mathrm{CH}$ NPs and TCH-NPs based upon the results of porosity, solgel fraction, swelling response, and drug release pattern. Physical properties of the NCHs such as swelling ratio, mesh size, porosity, swelling/deswelling nature, and diffusion coefficient, which may affect the release behavior of TG from the hydrogel matrix, can be adjusted and refined by controlling the hydrogel network's chemical structure. Nanocomposite hydrogels (NCHs) were successfully fabricated and loaded passively by TG containing $\mathrm{CH}-\mathrm{NPs}$ and TCH-NPs.

It was observed that the porosity of hydrogels increased by increasing monomer (AA) and polymer $(\mathrm{CH})$ concentration whereas it decreased by increasing crosslinker (MBAA) content. It has been reported that suitable porosity for hydrogel formulations should be around $90 \%$, for the majority of required applications. High porosity allows efficient uptake of fluids and gaseous exchange for cell attachment and sufficient drug loading. Sol fraction reflects unreactive reactants, ie, monomer, polymer, and crosslinker having aqueous solubility, whereas gel fraction displays grafting magnitude and crosslinking density. Likewise, gel fraction increased by increasing $\mathrm{CH}, \mathrm{AA}$, and MBAA concentrations, due to high crosslinking density.

Swelling studies demonstrated significant swelling behavior at higher alkaline $\mathrm{pH}$ (6.8 and 7.4) as compared to that at acidic $\mathrm{pH}$ of 1.2. At acidic $\mathrm{pH}$ (1.2), carboxylic acid groups found in AA chains were intact and protonated due to which there were reduced repulsive forces, hydrogen bonding predominated resulting in less water diffusion that ultimately decreased swelling. Swelling increased by increasing $\mathrm{pH}$ due to electrostatic repulsive forces pertaining among the anionic groups (-COO) of AA chains due to deprotonation of $\mathrm{COOH}$ groups. Due to the absence of hydrogen bonding and loose structure, more diffusion occurred resulting in increased swelling of hydrogel systems. At higher $\mathrm{pH}$ values, greater swelling behavior was

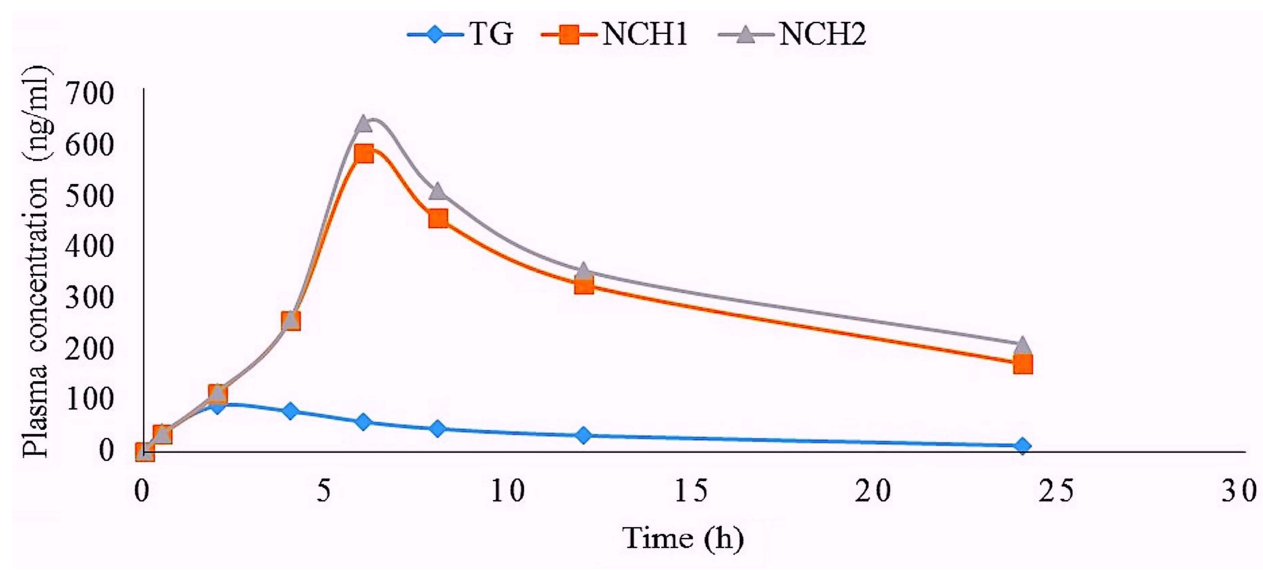

Figure 15 Plasma concentration-time profile of TG after administering the equivalent oral dose of $10 \mathrm{mg} / \mathrm{kg}$ in rabbits for pure ticagrelor and nanocomposite hydrogel formulations.

Abbreviations: TG, ticagrelor; $\mathrm{NCHI}$ and $\mathrm{NCH} 2$, nanocomposite hydrogel formulations. 
observed by increasing polymer $(\mathrm{CH})$ concentration, due to the availability of more grafting sites for polymerization. At alkaline $\mathrm{pH}$ repulsive forces dominated due to the presence of anionic groups. Formulation $\mathrm{H} 3(0.7 \% \mathrm{CH})$ displayed a higher swelling ratio, $\mathrm{q}$, and $\% \mathrm{ES}$ as shown in Figures $9 \mathrm{~A}$ and $10 \mathrm{~A}$. $\mathrm{CH}$ is a weak polyelectrolyte with a pKa of about 6.5. The primary $\mathrm{NH}_{2}$ group of the polymer $\mathrm{CH}$ undergoes protonation at $\mathrm{pH} 1.2$ while it gets deprotonated at $\mathrm{pH}$ 7.4, respectively. At low $\mathrm{pH}$ (1.2), primary amino groups of $\mathrm{CH}$ get protonated by $\mathrm{H}^{+}$ions and form $\mathrm{NH}_{3}{ }^{+}$groups that allow $\mathrm{CH}$ to form ionic linkages with sulfonate groups of KPS. Similarly, in an acidic environment, a screening effect of counter ions shields positively charged ammonium cations, preventing repulsion among them and ultimately reducing swelling capability. Both swelling ratio, $\mathrm{q}$, and \%ES increased significantly upon incrementing AA content, from $\mathrm{H} 4$ to $\mathrm{H} 6$, as represented in Figures 9B and 10B. This might be attributed to the fact that by increasing the concentration of AA acid, electrostatic forces of repulsion increased due to deprotonation of pendant $\mathrm{COOH}$ groups of $\mathrm{AA}$ that weakened hydrogen bonding, resulting in increased hydrogel swelling due to increased hydration. Moreover, by increasing AA concentration, enhanced water absorbency has been noted as it increases the diffusion of KPS across the pure $\mathrm{CH}$ backbone. Hence, it can be said that increasing concentration from $10 \%$ to $15 \%$ is optimal for achieving the maximum swelling ratio in the currently developed polymeric network. Similar results were reported by Wang et al for delivery of meloxicam and amoxicillin, where swelling of the co-polymer increased upon increasing monomer (AA) content. These results comply with the sol-gel fraction analysis that displayed a high gel content upon the incremental increase in the MBAA ratio. Hence, these copolymers emerged as stimuli-responsive as they displayed $\mathrm{pH}$-responsive swelling and drug release. The $\mathrm{pH}-$ responsive behavior will be beneficial if less drug is released at low gastric $\mathrm{pH}$, while more drug is released at high intestinal $\mathrm{pH}$ in a sustained and controlled manner, increasing the drug's residence time leading towards enhanced permeation and absorption. Similar findings were reported previously by Malik et al (2020) who prepared $\mathrm{CH} /$ Xanthan gum-based hydrogels to deliver an antiviral drug and observed increased swelling at higher $\mathrm{pH}$ values owing to reduced ionic repulsions. ${ }^{16,21}$

The IR pattern of TG displayed characteristic absorption peaks at $3403,3288,2933,2854,1605,1558,1505,1455$, $1316,1274,1256,1210,1110,1091$, and $761 \mathrm{~cm}^{-1}$ as presented in Figure 4. The absorption bands at 3403 and $3288 \mathrm{~cm}^{-1}$ specified the presence of -NH stretch and -OH stretch, while the peaks at 2933 and $2854 \mathrm{~cm}^{-1}$ pointed to the presence of alkyl stretch (-CH). The absorption bands at 1605 and $1558 \mathrm{~cm}^{-1}$ reflected the presence of $-\mathrm{N}-\mathrm{H}$ stretch, whereas the peaks at $1455 \mathrm{~cm}^{-1}$ indicated a methyl bend, at 1256 and $1210 \mathrm{~cm}^{-1}$ showed $-\mathrm{C}-\mathrm{OH}$ stretch. The intense peaks at 1110 and $1091 \mathrm{~cm}^{-1}$ signaled the presence of $-\mathrm{C}-\mathrm{O}$ stretch. Hydrogel formulations reflected distinctive peaks at 1710 , $1566,1550,1408,1323,1061,1018$, and $893 \mathrm{~cm}^{-1}$ indicating structural modifications during hydrogel formation. The peak at $1710 \mathrm{~cm}^{-1}$ refers to carbonyl stretching $(\mathrm{C}=\mathrm{O})$, whereas absorption bands at 1566 and $1550 \mathrm{~cm}^{-1}$ corresponded to the amide stretch $(-\mathrm{N}-\mathrm{H})$. The peak at $1323 \mathrm{~cm}^{-1}$ displayed -SH stretch while at 1061 and $1018 \mathrm{~cm}^{-1}$ showed -OH stretching. Moreover, characteristic absorption bands as observed in thiolated chitosan $(\mathrm{TCH})$ have also been seen in $\mathrm{NCH} 2$ containing TG-NPs. No other chemical interactions were observed between TG and polymer, reflecting efficient loading of TG entrapped CH-NPs and TC-NPs in NCH1 and NCH2, respectively. Similar shifts and overlaps have been reported by Wang et al, indicating grafting of monomer on the $\mathrm{CH}$ network through intermolecular as well as electrostatic interactions among various constituents. ${ }^{21}$

Morphological analysis revealed that unloaded hydrogels elaborated a porous and rough surface, whereas, SEM micrographs of $\mathrm{NCH} 1$ and $\mathrm{NCH} 2$ showed characteristic surface morphology with porous channels and cavities, and thus confirmed the distribution of extremely small-sized TG loaded nanoparticles throughout the hydrogel matrix (Figure 11). As reported earlier, increased content of $\mathrm{CH}$, the structure-forming component, increased the roughness scale of the hydrogel surface and promoted the porosity of the hydrogel network. This porous architecture and interconnected channels, as observed in NCH networks, is extremely benefic playing a pivotal role in swelling-deswelling kinetics. Furthermore, this porous structure will help in adhering and distributing the drug throughout the hydrogel network facilitating the uptake of biological fluids due to amplified capillary effect and increased surface area. This will ultimately facilitate the release of incorporated TG from the interpenetrating channels as well. Unlike conventional hydrogels, the porous hydrogel network having sponge-like characteristics can rapidly squeeze and expand, and absorb water in a wet state. Due to their porous nature and hydrated structure, hydrogels mimic native tissue surroundings. Mahmood et al revealed similar outcomes upon SEM analysis of 
nanocomposite hydrogels displaying voids and distribution channels throughout the developed hydrogel matrix. ${ }^{22}$

In XRD patterns of DLH, NCH1 and, $\mathrm{NCH} 2$, the peak intensities demonstrated by TG were greatly reduced indicating their amorphous nature (Figure 12). The characteristic diffraction peaks demonstrated by thiolated chitosan-based TC-NPs containing TG reflected the crystalline nature of formed nanoparticles, but with reduced peak intensities. TG displayed high-intensity peaks indicating its crystalline nature as depicted by its retarded dissolution. However, in the PXRD analysis of all hydrogel formulations, prominent structural changes have been depicted from a reduction of peak intensities. The sharp diffraction peaks were substituted by denser low-intensity peaks, demonstrating a decline in crystalline nature, possibly due to monomer and polymer conjugation. Furthermore, upon the comparison of peak intensities among the three hydrogel formulations, $\mathrm{NCH} 2$ depicted slightly higher peak intensities in the range of $18^{\circ}$ to $23^{\circ}$, as compared to those of DLH and NCH1. DLH and $\mathrm{NCH} 1$ displayed an overlapping peak pattern comprised of low-intensity peaks. No other interfering peaks corresponding to impurities were detected in the nanocomposite formulations. A similar consequence was observed by Shabir et al, for XRD of CH-co-AA and CH-co-MAA hydrogels. ${ }^{23}$

DSC thermogram of pure TG displayed a sharp endothermic peak at $142{ }^{\circ} \mathrm{C}$, corresponding to its melting point (MP) and reflecting its crystalline nature due to a high MP. A second exothermic peak was depicted at $320{ }^{\circ} \mathrm{C}$ reflecting crystallization of $\mathrm{TG}$ (Figure 5). Moreover, $\mathrm{NCH} 2$ displayed merging of TG's melting peak indicating structural transformation from crystalline character towards amorphous configuration. $\mathrm{NCH} 2$ displayed the first endothermic peak near $120{ }^{\circ} \mathrm{C}$ indicating possible interactions among $\mathrm{CH}$ and $\mathrm{TG}$ and the second broadened endothermic curve was observed near $200{ }^{\circ} \mathrm{C}$ followed by several small endothermic peaks that were also observed in the physical mixture. Morphological modifications occurred due to the polymerization of $\mathrm{CH}$ with other components resulting in an entirely different structural profile. Overall, thermal decomposition and weight loss of the $\mathrm{NCH}$ network was less as compared to pure TG and other components, significantly enhancing the stability of originated $\mathrm{NCH}$ networks. These results were in agreement with those obtained from the XRD analysis. A shift towards elevated glass transition temperature as displayed by $\mathrm{NCH}$ reflected excessive thermal stability of the composite network along with its biocompatibility and toughness and rigidity, predominantly due to high intermolecular interactions. Tummala et al, reported the same findings upon DSC analysis reflecting better biocompatibility among various components. $^{24}$

It was observed that drug entrapment efficiency and loading capacity of hydrogels increased with increasing concentration of $\mathrm{CH}$ from $0.3 \%$ to $1 \%$ and $\mathrm{AA}$ from $10 \%$ to $15 \%$. The reason behind this might be the enhanced efficiency of the polymer $(\mathrm{CH})$ to encapture more drug molecules thus improving the loading capacity. However, a decrease in the amount of drug-loaded in hydrogels has been observed when the concentration of cross-linker MBAA has been increased. The possible reason might be the greater cross-linking density that reduced the polymeric structure's elasticity, restricting TG movement into the newly developed hydrogel network, leading towards reduced drug entrapment efficiency.

Figure 13 demonstrates the drug release behavior of various hydrogel formulations. It was observed that $\% \mathrm{DR}$ was modified by varying ratios of polymer $(\mathrm{CH})$, monomer (AA) and cross-linker (MBAA). By increasing $\mathrm{CH}$ concentration from $0.3 \%$ to $1 \%$, drug release was observed to be enhanced over 24 hours. H1, H2, H3, and H6 showed an increasing trend of TG release from 80.12, 84.21, 90.32, to $92.95 \%$ respectively. Similarly, by increasing the AA (monomer) ratio in $\mathrm{H} 4$ to $\mathrm{H} 6, \% \mathrm{DR}$ was seen to be increased. $\% \mathrm{DR}$ of H4, H5, and H6 for 24 hours was found to be 74.23, 86.92, and $92.95 \%$ respectively. Moreover, formulations having varying concentrations of MBAA, \%DR was noticed to be decreased by increasing cross-linker (MBAA) content. H7, $\mathrm{H} 8$, and $\mathrm{H} 9$ displayed a cumulative release of $88.05,80.39$, and $71.4 \%$ respectively. It might be due to increased crosslinking density due to high MBAA content that eventually decreased the polymer network's elasticity. Among various formulations, H6 showed a maximum release of $92.95 \%$ probably due to its high $\mathrm{CH}$ and AA content that resulted in maximizing $\mathrm{pH}$ sensitivity and swelling ratio. \%DR was observed to be $72.3 \%$ and $76.5 \%$ after 24 hours for $\mathrm{NCH} 1$ and $\mathrm{NCH} 2$, respectively and is represented in Figure 13D. Hence, the reason for slow and sustained TG release might be that in the case of $\mathrm{NCH}$, the drug has to overcome two different barriers resulting in a prolonged effect much better than that of hydrogels or NPs alone. Gao et al developed thermosensitive hydrogels carrying norcantharidin NPs and doxorubicin and displayed similar drug release behavior by nanocomposites. $^{25}$

Kinetic modeling was established by comparing the value of $\mathrm{R}^{2}$ and it was found that data fits best in the Korsmeyer-Peppas model. This model describes the drug 
release mechanism ie Fickian or non-Fickian diffusion. As observed from the analysis, a value of $\mathrm{n}$ greater than 0.43 but less than $0.89(0.43<\mathrm{n}<0.89)$, indicated anomalous drug release by both diffusion and selling. Hence, the value of $\mathrm{n}$ indicated drug release following non-Fickian drug diffusion. Hence, drug release was observed to be in a controlled manner that occurred mainly through diffusion. $\mathrm{CH} /$ Xanthan gum hydrogels fabricated by Malik et al exhibited similar kinetics as displayed by currently developed hydrogel networks. ${ }^{16}$

A histopathological investigation of vital organs' tissue sections displayed no significant variation in their histopathology among all groups as no signs of inflammation, abnormality or lesions were detected. Neurons were intact and no sign of neuronal or axonal damage was noticed. The lungs showed normal alveolar epithelial cells with no signs of fluid accumulation or inflammation. No signs of cardiac ischemia were discerned. Moreover, the size of cardiomyocytes was found to be normal. Hepatocytes were normal with slight ballooning of cytoplasm as an indicator of cellular stress, no abnormality of sinusoidal places, macrophages were present in the sinusoidal spaces. Overall liver tissue showed no abnormality. On examination of the kidneys, glomerulus was found normal and connected with the bowmen capsule. Accumulation of immune cells was not detected along with no indication of tubular or ductal abnormality. Stomach tissue was normal, no sign of gastric ulceration was spotted. Epithelial cells were normal as there was no sign of hyperplasia. Intestinal Brush Border cells were intact. No sign of hyperplasia or cellular damage, immune cells accumulation, or ulceration was observed. Conclusively, no vital differences upon histopathological investigation were discerned between the treatment groups and control group attributing reason to the safe use of $\mathrm{NCHs}$ for TG delivery. Hence, an oral dose level up to $5 \mathrm{~g} / \mathrm{kg}$ body weight of the developed nanocomposite network was well tolerated in the whole 14 day study period, indicating that the developed nanocomposite hydrogels are non-toxic. Acute oral toxicity studies were also conducted previously upon $\beta$ CD-g-MAA and montmorilonite nanocomposite hydrogels by Mahmood et al, PVA and itaconic acid-co-MAA hydrogel networks by Abdullah et al, and upon $\mathrm{CH}$ and Xanthan gumbased hydrogels by Malik et al. Results displayed by NCHs were comparable to those reported by these researchers. Thus, NCHs can be used safely for oral delivery of TG due to their non-toxic nature. ${ }^{16,22,26}$

The pharmacokinetic evaluation revealed that $\mathrm{C}_{\max }$ of both $\mathrm{NCH} 1$ and $\mathrm{NCH} 2$ showed a 6.5 and 7.2 fold increment, respectively. $\mathrm{T}_{\max }$ increased from $2 \mathrm{hrs}$ (pure TG) to $6 \mathrm{hrs}$ for both $\mathrm{NCH}$ formulations. Similarly, half-life and $\mathrm{AUC}_{0-\infty}$ of $\mathrm{NCH} 1$ and $\mathrm{NCH} 2$ was observed to be increased remarkably when compared with that of pure TG. The half-life of NCH1 increased up to $11.6 \mathrm{hrs}$ and that of $\mathrm{NCH} 2$ prolonged for up to $13.09 \mathrm{hrs}$, whereas $\mathrm{t}_{1 / 2}$ of pure TG was found to be equivalent to $7.73 \mathrm{hrs}$. $\mathrm{AUC}_{0-\infty}$ of $\mathrm{NCH} 1$ and $\mathrm{NCH} 2$ was observed to be 9789.42 and $11,620.74 \mathrm{ng} / \mathrm{mL}$ respectively, very significant as compared to pure TG's area under the curve (1034.72 ng/ $\mathrm{mL}$ ). Moreover, relative bioavailability (\%RBA) was enhanced considerably in both $\mathrm{NCH}$ formulations, probably due to enhanced mucoadhesion, improved permeability, and hence prolonging contact time that ultimately resulted in ameliorated oral bioavailability. Nanoparticles might have played a positive role in increasing the transcellular and paracellular transport of TG due to their extremely small size. Son et al fabricated TG-loaded nano lipid carrier systems for enhancing the oral bioavailability of TG. NLCs demonstrated a relative bioavailability of $254.9 \%$ upon comparison with pure TG based upon increased AUC, but $\mathrm{T}_{\max }$ was noticed to be reduced (1.2 h) as compared to pure TG's $\mathrm{T}_{\max }(2.6 \mathrm{~h})$. Whereas, NCHs displayed a slow and sustained release as displayed and confirmed from prolongation of half-life and prolonged duration of action as depicted by increment in the $\mathrm{T}_{\max }$ value $(6 \mathrm{~h}) .{ }^{14} \mathrm{Na}$ et al prepared self micro emulsifying drug delivery system for oral delivery of TG and displayed a reduction in $\mathrm{T}_{\max }$ to $0.75 \mathrm{~h}$ and elevated $\%$ RBA of TG to $637 \%$. $^{12}$

Similar drug delivery systems based upon nanocomposite networks have been reported by some other researchers as well. Gao et al designed an in situ thermoresponsive hydrogel network by thin-film dispersion method for delivery of norcantharidin based NPs and doxorubicin for intratumoral administration. These NCHs exhibited biodegradability along with safe and effective drug delivery. ${ }^{25}$ Dos Santos et al, formulated transparent $\mathrm{CH} / \mathrm{PLGA}$ nanoparticles composite hydrogels encapsulating an enriched flavonoid fraction of Cecropia glaziovii Snethl by directly incorporating NPs at different concentrations ( $1 \%$ to $10 \% \mathrm{w} / \mathrm{w}$ ) to gelling aqueous $\mathrm{CH}$ solutions. ${ }^{34}$ Eivazzadeh-Keihan et al fabricated a nano biocomposites scaffold by incorporating polyvinyl alcohol, sodium alginate, silk fibroin, and $\mathrm{Mg}(\mathrm{OH})_{2}$ nanorods, possessing vast applications such as a scaffold for being used in tissue engineering and wound dressings, as well as an efficient drug delivery system due to having substantial biological activities. ${ }^{27}$ In the current study, TG was efficiently loaded in the form of CH-NPs and TC-NPs and formulated in the form of nanocomposite hydrogels that displayed 
a sustained release due to prolongation of half-life along with enhanced oral bioavailability, ameliorating the advantages of both hydrogels' stability and biocompatibility as well as the nanoparticles' effective and safe drug delivery. Some other additional advantages displayed by $\mathrm{NCH}$ formulation are its biodegradability, $\mathrm{pH}$ responsiveness, non-toxic nature, and prolonged duration of action. Hence NCHs can be effectively utilized for orally administering TG to enhance its effectiveness and antiplatelet activity.

\section{Conclusion}

Nanocomposite hydrogels (NCHs) were successfully fabricated and loaded with TG containing chitosan and thiolated chitosan-based nanoparticles, CH-NPs, and TC-NPs, respectively. Both $\mathrm{NCH}$ formulations $\mathrm{NCH} 1$ and $\mathrm{NCH} 2$ displayed $\mathrm{pH}$-dependent swelling followed by controlled drug release for 24 hours. Hence, both NCH formulations showed promising results for $\mathrm{pH}$-responsive delivery of TG. In vivo bioavailability studies demonstrated a higher relative bioavailability by $\mathrm{NCH} 2$ containing TC-NPs as compared to NCH1 containing CH-NPs. Moreover, TG's half-life and Tmax were prominently prolonged. Similarly, $\mathrm{C}_{\max }$ and AUC were also seen to be augmented. Therefore, nanocomposite hydrogel formulations can be effectively used for enhancing TG bioavailability and anticoagulant effect for a prolonged time and can help to increase patient compliance as well by reducing dosing frequency.

\section{Acknowledgment}

The authors extended their appreciation to the Distinguished Scientist Fellowship program at King Saud University, Riyadh, Saudi Arabia for funding this work through Research Supporting Project Number (RSP-2021/131).

\section{Disclosure}

No potential conflict of interest for this work was reported by the authors.

\section{References}

1. Maher S, Brayden DJ, Casettari L, Illum L. Application of permeation enhancers in oral delivery of macromolecules: an update. Pharmaceutics. 2019;11(1):41. doi:10.3390/pharmaceutics11010041

2. Ibrahim YHY, Regdon G, Hamedelniel EI, Sovány T. Review of recently used techniques and materials to improve the efficiency of orally administered proteins/peptides. DARU. 2020;28(1):403-416. doi:10.1007/s40199-019-00316-w

3. Dannert C, Stokke BT, Dias RS. Nanoparticle-hydrogel composites: from molecular interactions to macroscopic behavior. Polymers. 2019;11(2):275. doi:10.3390/polym 11020275
4. Kale AR, Kakade S, Bhosale AA. Review on: solubility enhancement techniques. J Curr Pharm Res. 2020;10(2):3630-3647.

5. Li X, Su X. Multifunctional smart hydrogels: potential in tissue engineering and cancer therapy. J Mater Chem B. 2018;6 (29):4714-4730. doi:10.1039/C8TB01078A

6. Zhao F, Yao D, Guo R, Deng L, Dong A, Zhang J. Composites of polymer hydrogels and nanoparticulate systems for biomedical and pharmaceutical applications. Nanomaterials. 2015;5(4):2054-2130. doi:10.3390/nano5042054

7. Gaharwar AK, Peppas NA, Khademhosseini A. Nanocomposite hydrogels for biomedical applications. Biotechnol Bioeng. 2014;111 (3):441-453. doi:10.1002/bit. 25160

8. Teng R, Maya J. Absolute bioavailability and regional absorption of ticagrelor in healthy volunteers. J Drug Assess. 2014;3(1):43-50. doi:10.3109/21556660.2014.946604

9. Esquivel R, Juárez J, Almada M, Ibarra J, Valdez MA. Synthesis and characterization of new thiolated chitosan nanoparticles obtained by ionic gelation method. Int J Polym Sci. 2015;2015:1-18. doi:10.1155/2015/ 502058

10. Ahmed TA, Aljaeid BM. Preparation, characterization, and potential application of chitosan, chitosan derivatives, and chitosan metal nanoparticles in pharmaceutical drug delivery. Drug Des Devel Ther. 2016;10:483. doi:10.2147/DDDT.S99651

11. Fan B, Xing Y, Zheng Y, Sun C, Liang G. pH-responsive thiolated chitosan nanoparticles for oral low-molecular weight heparin delivery: in vitro and in vivo evaluation. Drug Deliv. 2016;23(1):238-247. doi:10.3109/10717544.2014.909908

12. Na Y-G, Byeon -J-J, Wang M, et al. Strategic approach to developing a self-microemulsifying drug delivery system to enhance antiplatelet activity and bioavailability of ticagrelor. Int $J$ Nanomedicine. 2019;14:1193. doi:10.2147/IJN.S190426

13. Son G-H, Na Y-G, Huh HW, et al. Systemic design and evaluation of ticagrelor-loaded nanostructured lipid carriers for enhancing bioavailability and antiplatelet activity. Pharmaceutics. 2019;11(5):222. doi:10.3390/pharmaceutics11050222

14. Othman N, Masarudin MJ, Kuen CY, Dasuan NA, Abdullah LC. Synthesis and optimization of chitosan nanoparticles loaded with L-ascorbic acid and thymoquinone. Nanomaterials. 2018;8(11):920. doi:10.3390/nano8110920

15. Nguyen TV, Nguyen TTH, Wang S-L, Vo TPK, Nguyen AD. Preparation of chitosan nanoparticles by TPP ionic gelation combined with spray drying, and the antibacterial activity of chitosan nanoparticles and a chitosan nanoparticle-amoxicillin complex. Res Chem Intermed. 2017;43(6):3527-3537. doi:10.1007/s11164-016-2428-8

16. Malik NS, Ahmad M, Minhas MU, et al. Chitosan/xanthan gum based hydrogels as potential carrier for an antiviral drug: fabrication, characterization, and safety evaluation. Front Chem. 2020;8:50. doi:10.3389/fchem.2020.00050

17. Nawaz S, Khan S, Farooq U, et al. Biocompatible hydrogels for the controlled delivery of anti-hypertensive agent: development, characterization and in vitro evaluation. Des Monomers Polym. 2018;21 (1):18-32. doi:10.1080/15685551.2018.1445416

18. OECD. Guidance Document on Acute Oral Toxicity Testing. Paris, France: OECD; 2000.

19. Ni XL, Chen LX, Zhang H, et al. In vitro and in vivo antitumor effect of gefitinib nanoparticles on human lung cancer. Drug Deliv. 2017;24 (1):1501-1512. doi:10.1080/10717544.2017.1384862

20. Bueno LM, Manoel JW, Giordani CFA, et al. HPLC method for simultaneous analysis of ticagrelor and its organic impurities and identification of two major photodegradation products. Eur J Pharm Sci. 2017;97:22-29. doi:10.1016/j.ejps.2016.11.004

21. Wang Q, Xie X, Zhang X, Zhang J, Wang A. Preparation and swelling properties of $\mathrm{pH}$-sensitive composite hydrogel beads based on chitosan-g-poly (acrylic acid)/vermiculite and sodium alginate for diclofenac controlled release. Int J Biol Macromol. 2010;46 (3):356-362. doi:10.1016/j.ijbiomac.2010.01.009 
22. Mahmood A, Amara Sharif FM, Sarfraz RM, et al. Development and in vitro evaluation of ( $\beta$-cyclodextrin-g-methacrylic acid)/Na+montmorillonite nanocomposite hydrogels for controlled delivery of lovastatin. Int J Nanomedicine. 2019;14:5397. doi:10.2147/IJN.S209662

23. Shabir F, Erum A, Tulain UR, Hussain MA, Ahmad M, Akhter F. Preparation and characterization of $\mathrm{pH}$ sensitive crosslinked linseed polysaccharides-co-acrylic acid/methacrylic acid hydrogels for controlled delivery of ketoprofen. Des Monomers Polym. 2017;20 (1):485-495. doi:10.1080/15685551.2017.1368116

24. Tummala S, Kumar MS, Prakash A. Formulation and characterization of 5-fluorouracil enteric coated nanoparticles for sustained and localized release in treating colorectal cancer. Saudi Pharm J. 2015;23 (3):308-314. doi:10.1016/j.jsps.2014.11.010

25. Gao B, Luo J, Liu Y, et al. Intratumoral administration of thermosensitive hydrogel co-loaded with norcantharidin nanoparticles and doxorubicin for the treatment of hepatocellular carcinoma. Int J Nanomedicine. 2021;16:4073-4085. doi:10.2147/IJN.S308057

26. Abdullah O, Usman Minhas M, Ahmad M, Ahmad S, Barkat K, Ahmad A. Synthesis, optimization, and evaluation of polyvinyl alcohol-based hydrogels as controlled combinatorial drug delivery system for colon cancer. Adv Polym Technol. 2018;37 (8):3348-3363. doi:10.1002/adv.22119

27. Eivazzadeh-Keihan R, Khalili F, Aliabadi HAM, et al. Alginate hydrogel-polyvinyl alcohol/silk fibroin/magnesium hydroxide nanorods: a novel scaffold with biological and antibacterial activity and improved mechanical properties. Int $J$ Biol Macromol. 2020;162:1959-1971. doi:10.1016/j.ijbiomac.2020.08.090

28. Eivazzadeh-Keihan R, Radinekiyan F, Maleki A, Bani MS, Hajizadeh Z, Asgharnasl S. A novel biocompatible core-shell magnetic nanocomposite based on cross-linked chitosan hydrogels for in vitro hyperthermia of cancer therapy. Int $J$ Biol Macromol. 2019;140:407-414. doi:10.1016/j.ijbiomac.2019.08.031
29. Mohamed NA, El-Ghany NAA, Fahmy MM. Novel antimicrobial superporous cross-linked chitosan/pyromellitimide benzoyl thiourea hydrogels. Int J Biol Macromol. 2016;82:589-598. doi:10.1016/j. ijbiomac.2015.09.023

30. Singh J, Srivastava M, Dutta J, Dutta P. Preparation and properties of hybrid monodispersed magnetic $\alpha$-Fe2O3 based chitosan nanocomposite film for industrial and biomedical applications. Int $\mathrm{J} \mathrm{Biol}$ Macromol. 2011;48(1):170-176. doi:10.1016/j.ijbiomac.2010.10.016

31. Aliabadi M, Irani M, Ismaeili J, Najafzadeh S. Design and evaluation of chitosan/hydroxyapatite composite nanofiber membrane for the removal of heavy metal ions from aqueous solution. J Taiwan Inst Chem Eng. 2014;45(2):518-526. doi:10.1016/j.jtice.2013.04.016

32. Eivazzadeh-Keihan R, Dogari H, Ahmadpour F, et al. Design and synthesis of a novel nanocomposite based on magnetic dopamine nanoparticles for purification of $\alpha$-amylase from the bovine milk. Sci Rep. 2021;11(1):1-15. doi:10.1038/s41598-021-92919-0

33. Widjaja LK, Bora M, Chan PNPH, Lipik V, Wong TT, Venkatraman SS. Hyaluronic acid-based nanocomposite hydrogels for ocular drug delivery applications. J Biomed Mater Res A. 2014;102(9):3056-3065. doi:10.1002/jbm.a.34976

34. Dos Santos TC, Hernández R, Rescignano N, et al. Nanocomposite chitosan hydrogels based on PLGA nanoparticles as potential biomedical materials. Eur Polym J. 2018;99:456-463. doi:10.1016/j. eurpolymj.2017.12.039
International Journal of Nanomedicine

\section{Publish your work in this journal}

The International Journal of Nanomedicine is an international, peerreviewed journal focusing on the application of nanotechnology in diagnostics, therapeutics, and drug delivery systems throughout the biomedical field. This journal is indexed on PubMed Central, MedLine, CAS, SciSearch ${ }^{\mathbb{}}$, Current Contents ${ }^{\mathbb{R}} /$ Clinical Medicine,

\section{Dovepress}

Journal Citation Reports/Science Edition, EMBase, Scopus and the Elsevier Bibliographic databases. The manuscript management system is completely online and includes a very quick and fair peer-review system, which is all easy to use. Visit http://www.dovepress.com/ testimonials.php to read real quotes from published authors. 